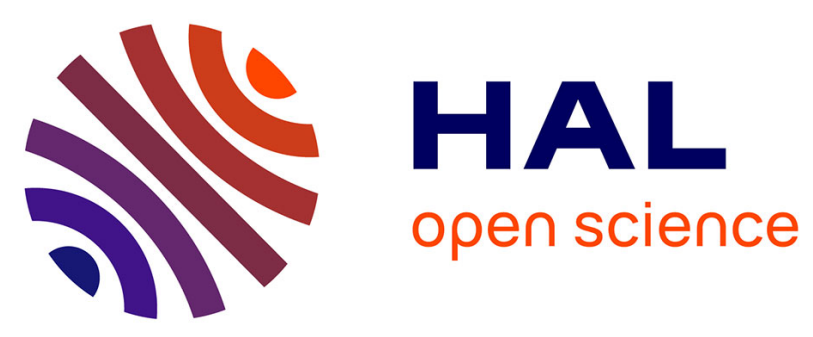

\title{
Engraissement des vaches de réforme de race Charolaise: Facteurs de variation des performances zootechniques, de la composition tissulaire des carcasses et de la qualité organoleptique de la viande
}

Rémi Dumont, M. Roux, Jacques Agabriel, C. Touraille, J. Bonnemaire, C.

Malterre, J. Robelin

\section{To cite this version:}

Rémi Dumont, M. Roux, Jacques Agabriel, C. Touraille, J. Bonnemaire, et al.. Engraissement des vaches de réforme de race Charolaise: Facteurs de variation des performances zootechniques, de la composition tissulaire des carcasses et de la qualité organoleptique de la viande. Productions Animales, 1991, 4 (4), pp.271-286. hal-00895946

\section{HAL Id: hal-00895946 \\ https://hal.science/hal-00895946}

Submitted on 1 Jan 1991

HAL is a multi-disciplinary open access archive for the deposit and dissemination of scientific research documents, whether they are published or not. The documents may come from teaching and research institutions in France or abroad, or from public or private research centers.
L'archive ouverte pluridisciplinaire HAL, est destinée au dépôt et à la diffusion de documents scientifiques de niveau recherche, publiés ou non, émanant des établissements d'enseignement et de recherche français ou étrangers, des laboratoires publics ou privés. 
INRA Prod. Anim., 1991, 4 (4), $271-286$
DUMONT R., ROUX M., AGABRIEL J. *, TOURAILLE $C .^{* *}$, BONNEMAIRE J., MALTERRE $C .{ }^{*}$ et ROBELIN J. *

INRA - ENSSAA

Laboratoire de la Chaire de Zootechnie 26, boulevard Docteur Petitjean 21000 Dijon

* INRA Laboratoire Croissance et Métabolismes des Herbivores Theix 63122 Saint-Genès-Champanelle

* INRA Station de Recherches sur la Viande

Theix 63122 Saint-Genès-Champanelle

\section{Engraissement des vaches de réforme de race Charolaise}

\section{Facteurs de variation des performances zootechniques, de la composition tissulaire des carcasses et de la qualité organoleptique de la viande}

\author{
Dans certains systèmes de production Charolais, la vente des vaches \\ de réforme grasses contribue pour 20 à $30 \%$ au produit brut de l'élevage. \\ Mieux maîtriser les conditions de finition de ces animaux peut être \\ d'un grand intérêt pour une bonne valorisation bouchère des carcasses \\ et pour l'amélioration des qualités organoleptiques de la viande.
}

\section{Résumé}

Les conditions de finition de la vache de réforme de race Charolaise ont été étudiées au cours de 2 essais portant sur 67 vaches : 11 abattues maigres et 56 engraissées avec un régime à hase d'ensilage de maïs. Ils ont permis de mettre en évidence l'influence de la durée d'engraissement (0,70 ou 109 jours), de l'âge moyen (5 ou 11 ans) et d'un implant d'acétate de trenbolone sur la reprise de poids, la capacité d'ingestion, la composition tissulaire des carcasses, la proportion de muscle dans le gain de poids, les caractéristiques physico-chimiques et les qualités organoleptiques de la viande.

Pour l'ensemble des vaches non implantées, le gain de poids vif se situe entre 1100 et $1200 \mathrm{~g} / \mathrm{j}$ pour une durée d'engraissement d'environ 2 mois et demi. Elles ingèrent en moyenne $13,7 \mathrm{~kg}$ de matière sèche par jour, leur efficacité alimentaire est de l'ordre de $90 \mathrm{~g}$ de croît par UFL ingérée. A l'abattage, ces animaux fournissent des carcasses dont le poids chaud moyen est de $385 \mathrm{~kg}$, composées de $66 \%$ de muscle, $19 \%$ de tissu adipeux et $15 \%$ d'os. Par rapport aux vaches abattues maigres, le gain de carcasse au cours de l'engraissement est de $63 \mathrm{~kg}$ dont $37 \%$ sous forme de muscle.

La prolongation de l'engraissement jusqu'à 110 jours peut permettre d'accroître le poids de carcasse de 20 à $30 \mathrm{~kg}$ mais ce surplus de carcasse est essentiellement constitué de tissu adipeux.

Dans les conditions de cette expérimentation, il n'est pas possible de conclure à un effet négatif très net de l'âge des animaux sur les performances d'engraissement bien que le gain de poids vif, l'efficacité alimentaire et le rendement à l'abattage apparaissent plus faibles chez les animaux les plus âgés.

L'implant d'acétate de trenbolone a un effet marqué sur la reprise de poids des vaches : $57 \mathrm{~kg}$ supplémentaires de poids vif pour l'ensemble des animaux avec une prolongation de la durée d'engraissement de 27 jours. Il améliore également la proportion de tissu musculaire dans le gain de carcasse $(47 \%$ contre $41 \%$ pour le lot témoin chez les vaches âgées).

L'engraissement des carcasses (comparaison entre vache maigre et vache engraissée) avec pour corollaire l'augmentation de la teneur en lipides intramusculaires améliore très nettement la tendreté, la jutosité et la flaveur de la viande avec diminution de la force de cisaillement. Les animaux les plus âgés (11 ans), produisent une viande jugée plus dure à la dégustation alors qu'on n'observe pas d'augmentation significative avec l'âge de la force de cisaillement. Enfin cet essai ne met pas en évidence d'effet négatif de l'acétate de trenbolone sur les caractéristiques sensorielles et physico-chimiques du muscle.
En France, au cours des dix dernières années, le nombre total de vaches conduites en système allaitant spécialisé a fortement augmenté (+ $22 \%)$. Actuellement, la race Charolaise est la plus représentée dans ce type d'élevage avec $41 \%$ de l'effectif total de femelles soit 1430000 têtes (RGA 1988).

Dans les troupeaux Charolais, la vente de vaches de réforme contribue pour 20 à $30 \%$ au produit brut de l'élevage (Lherm et al 1988), mais avec de fortes variations selon le système de production et le «degré » de finition des vaches. Cependant on constate depuis quelques années une diminution de la production d'animaux gras dans ces élevages (CEREOPA 1987 ; ITEB 1989) et la vache de réforme ne semble pas échapper à ce phénomène.

Les pratiques d'engraissement des vaches de réforme sont très diversifiées selon les exploitations. Certains éleveurs essaient d'engraisser leurs animaux à l'herbe, mais cela nécessite de déceler très tôt les vaches vides. Généralement l'engraissement commence après le sevrage des veaux à l'automne et s'effectue à l'étable, sur une courte période, avec des rations ayant une concentration nutritive élevée.

Les principales difficultés rencontrées pour l'engraissement de ces animaux sont liées à leur hétérogénéité qui concerne l'âge, le format, l'état corporel, l'état sanitaire et l'état physiologique. Les effets de tels facteurs ont déjà été décrits en France chez des vaches de races laitières Normande, Française Frisonne et croisées [Béranger et Malterre 1968, Béranger et al 1970, Malterre 1972, Chilliard et al 1984, Colleau et al 1984] ou de races allaitantes Salers et Limousine (Malterre 1986, Malterre et al 1989) 
et à l'étranger pour des vaches de races Hereford et Angus (Swingle et al 1979, Matulis et al 1987) ou de race Holstein (Jones 1983). Une synthèse de ces résultats a été publiée par Malterre et Jones (1991).

La race Charolaise, qui se caractérise par un développement assez tardif et une faible adiposité, n'a pas fait l'objet jusqu'à présent de travaux analogues. C'est pour cette raison qu'un programme de recherche de références sur ce sujet a été mis en place à l'INRA.

Les essais ont été réalisés au domaine INRA de Dijon-Epoisses. L'objectif des deux premières études de ce programme (1986-88), présentées dans cet article, a été de décrire avec précision la finition des vaches de réforme avec un régime alimentaire bien connu à base d'ensilage de maïs, en étudiant les effets de la durée d'engraissement, de l'âge et d'une substance anabolisante sur la capacité d'ingestion, la reprise de poids, la composition tissulaire des carcasses, la part respective des tissus adipeux et musculaire dans le gain de poids et enfin les caractéristiques physico-chimiques du muscle et la qualité organoleptique de la viande.

\section{1 / Conditions expérimentales}

Les vaches ont été achetées à des domaines expérimentaux (INRA, CEMAGREF, ITEB, EDE), à des établissements d'enseignement agricole et à des groupements de producteurs. A l'achat, elles devaient correspondre aux caractéristiques suivantes : avoir réalisé au moins une lactation (1 à 3 pour les animaux jeunes, 6 à 10 pour les animaux âgés), être vides et taries après avoir allaité leur veau, être en bon état sanitaire et suffisamment maigres.

\section{1 / Schéma expérimental}

Deux essais factoriels successifs ont permis de tester les effets de la durée d'engraissement (essai 1), de l'âge et de l'administration d'un anabolisant (essai 2) sur les performances des animaux, la qualité des carcasses et de la viande. De plus, on a cherché à estimer la reprise de poids de carcasse et sa composition tissulaire. Pour cela, il a été nécessaire, d'une part, d'abattre des animaux avant engraissement et d'autre part, de procéder à des dissections complètes d'une demi-carcasse chez ces animaux maigres et chez leurs homologues une fois engraissés.

Dans l'essai 1, 27 vaches ont été réparties en 3 lots homologues: un lot (M) abattu «maigre » avant engraissement et deux lots (G1 et G2) engraissés respectivement pendant 70 jours (état d'engraissement jugé optimum) et 109 jours.

L'essai 2 a porté sur 40 vaches : 21 jeunes (J) ayant en moyenne 5 ans et 19 âgées (A) ayant en moyenne 11 ans. Chaque catégorie d'âge a été répartie en 3 lots : un lot maigre (JM et AM) abattu avant engraissement, un lot témoin (JT et AT) engraissé pendant 56 jours, et un lot implanté (JI et AI) avec $300 \mathrm{mg}$ d'acétate de trenbolone, engraissé jusqu'à atteindre le même état d'engraissement moyen que le lot témoin, soit 83 jours.

Pour chaque essai, les différents lots ont été constitués avant le début de la période expérimentale et étaient homologues sur la base $\mathrm{du}$ poids vif (double pesée), de l'âge, de l'état d'engraissement apprécié par maniements (note de 0 à 5 , Agabriel et al 1986) et par mesure du diamètre moyen des adipocytes du tissu adipeux sous-cutané caudal (Robelin et Agabriel 1986). De plus, dans chaque lot destiné à être engraissé, on a choisi, à la mise en lot, 3 à 5 animaux représentatifs de leur lot et homologues du lot maigre correspondant (tableau 1). Ces animaux après engraissement (à l'exception de ceux provenant du lot G1) et ceux abattus maigres ont fait l'objet d'une dissection complète de la demi-carcasse droite.

Tableau 1. Caractéristiques des animaux à la mise en lot.

\begin{tabular}{|c|c|c|c|c|c|c|c|c|c|}
\hline & \multicolumn{3}{|c|}{ Essai 1 } & \multicolumn{5}{c|}{ Essai 2 } \\
\hline Facteur de variation étudié & \multicolumn{3}{|c|}{$\begin{array}{c}\text { Durée } \\
\text { d'engraissement }\end{array}$} & \multicolumn{3}{c|}{ Age $\times$ Implant d'acétate de trenbolone } \\
\hline Nombre d'animaux & \multicolumn{3}{|c|}{27} & \multicolumn{5}{c|}{40} \\
\hline Lot & M & G1 & G2 & JM & JT & II \\
\hline Durée d'engraissement (j) & 0 & 70 & 109 & 0 & 56 & 83 & 0 & 56 & 83 \\
\hline Effectif / lot & 4 & 13 & 10 & 3 & 9 & 9 & 4 & 6 & 9 \\
\hline Poids vif (kg) & 607 & 596 & 583 & 611 & 593 & 603 & 626 & 652 & 658 \\
\hline Age (années) & 8,5 & 8,8 & 8,7 & 5,3 & 5,1 & 5,4 & 11,5 & 11,5 & 11,3 \\
\hline Note d'état (sur 5) & 1,5 & 1,6 & 1,7 & 1,5 & 1,5 & 1,6 & 1,1 & 1,3 & 1,7 \\
\hline Diamètre des adipocytes ( $\mu$ ) & 23 & 34 & 34 & 43 & 48 & 60 & 44 & 43 & 52 \\
\hline Nombre d'animaux & 4 & 0 & 5 & 3 & 4 & 4 & 4 & 4 & 4 \\
\hline
\end{tabular}




\section{2 / Déroulement des essais}

Les animaux sont arrivés en novembre. Une période préliminaire a permis d'effectuer les traitements sanitaires et d'homogénéiser les états d'engraissement, en limitant l'ingestion d'une ration foin-paille chez les animaux les plus gras (figure 1). Elle a été suivie d'une phase d'accoutumance au régime expérimental ; toutes les vaches ont alors reçu le même régime $(6 \mathrm{~kg}$ de matière sèche d'ensilage de maïs, $0,2 \mathrm{~kg}$ de tourteau de soja, $40 \mathrm{~g}$ d'urée et $150 \mathrm{~g}$ de complément minéral vitaminisé). La constitution des lots a eu lieu à la fin de la période préliminaire dans l'essai 2 et à la fin de la période d'accoutumance dans l'essai 1. Les animaux maigres ont été abattus au début de l'engraissement pour l'essai 1 et au début de la période d'accoutumance pour l'essai 2. Dans ce dernier cas, nous avons fait l'hypothèse que pendant la phase d'accoutumance, il y avait seulement une diminution du contenu digestif sans modification du poids vif vide ou du poids de carcasse.

L'engraissement a été réalisé en stabulation libre par case de 10 avec un régime à base d'ensilage de maïs distribué à volonté (tableau 2), en deux repas par jour, le concentré (tourteau de soja et pulpes de betteraves) étant distribué avant chaque repas. Au cours de la période expérimentale, les quantités ingérées d'ensilage de maïs ont été contrôlées par lot 5 jours par semaine. La valeur nutritive des aliments (tableau 2) a été estimée à partir des résultats de l'analyse chimique d'échantillons représentatifs des aliments distribués, complétés, pour l'ensilage de maïs de l'essai 2, par des mesures sur moutons de digestibilité de la matière organique. En outre, les vaches ont été pesées une fois par semaine ; une double pesée a eu lieu au début et à la fin de chaque période. Le gain de poids moyen a été estimé individuellement par régression linéaire simple ou quadratique.
Figure 1. Schéma expérimental et déroulement des essais.

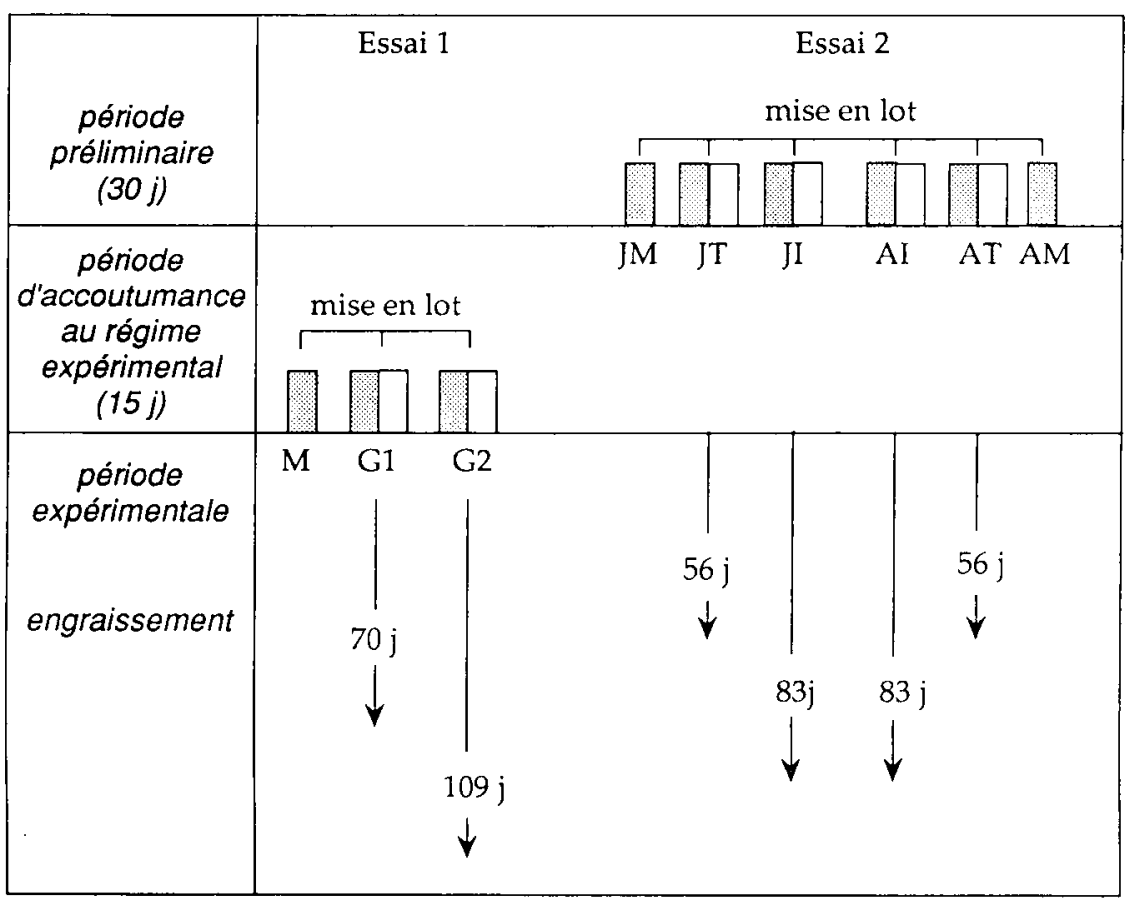

Lots homologues dont la demi-carcasse a été disséquée (sauf pour G1)

\section{3 / Mesures à l'abattage}

Toutes les vaches ont été abattues à l'abattoir expérimental de l'INRA de Theix. Les carcasses et les principaux éléments du 5" quartier ont été pesés séparément (dépôts adipeux notamment). La conformation des carcasses a été appréciée par mensurations selon Frebling et al (1967). Pour 32 animaux, la composition tissulaire de la carcasse a été déterminée par dissection en séparant au couteau les différents tissus (mus-

Tableau 2. Régimes alimentaires et valeur nutritive des ensilages de mais.

\begin{tabular}{|l|c|c|}
\hline & Essai 1 & Essai 2 \\
\hline Régimes distribués (par jour) & & \\
- Ensilage de maïs & A volonté & A volonté \\
- Tourteau de soja (kg) & 0,6 & 0,8 \\
- Pulpes de betteraves déshydratées (kg) & 1,5 & 1,3 \\
- Urée (kg) & 0,1 & 0,1 \\
- CMV (kg) & 0,15 & 0,15 \\
\hline Composition chimique des ensilages & & \\
de maïs & & 328 \\
- MS (g/kg) & 303 & 952 \\
- MO (g/kg MS) & 953 & 79 \\
- MAT (g/kg MS) & 77 & 184 \\
- CB (g/kg MS) & 196 & 69,7 \\
\hline Digestibilité mesurée sur moutons (\%) & & 71,5 \\
- CUD MS & - & \\
- CUD MO & - & 0,92 \\
\hline Valeur nutritive du maïs (/kg MS) & & 49 \\
- UFL & 0,85 & 68 \\
- PDIN (g) & 47 & 65 \\
- PDIE (g) & & \\
\hline
\end{tabular}


cles, os, dépôts adipeux) de la demi-carcasse droite. Le poids des différentes régions musculaires et des différents tissus adipeux (souscutané, intermusculaire, interne, omental, mésentérique et périrénal) ont également été mesurés. L'ensemble des tissus a été broyé séparément et a fait l'objet d'une analyse chimique (teneur en eau, lipides, protéines, minéraux et valeur calorifique) afin de déterminer la composition chimique du corps entier. Les résultats détaillés de ces mesures ont fait l'objet d'une publication spécifique (Robelin et al 1990).

Pour tous les animaux, la composition de la carcasse a également été estimée à partir des résultats de dissection de la $6^{\circ}$ côte, du poids des dépôts adipeux du $5^{\mathrm{e}}$ quartier et des quatre os canons (Robelin, non publié).

\section{4 / Analyses physico-chimiques et sensorielles de la viande}

Tous les muscles étudiés ont été prélevés sur les carcasses 24 heures environ post mortem. Pour le ressuage, les carcasses ont été laissées

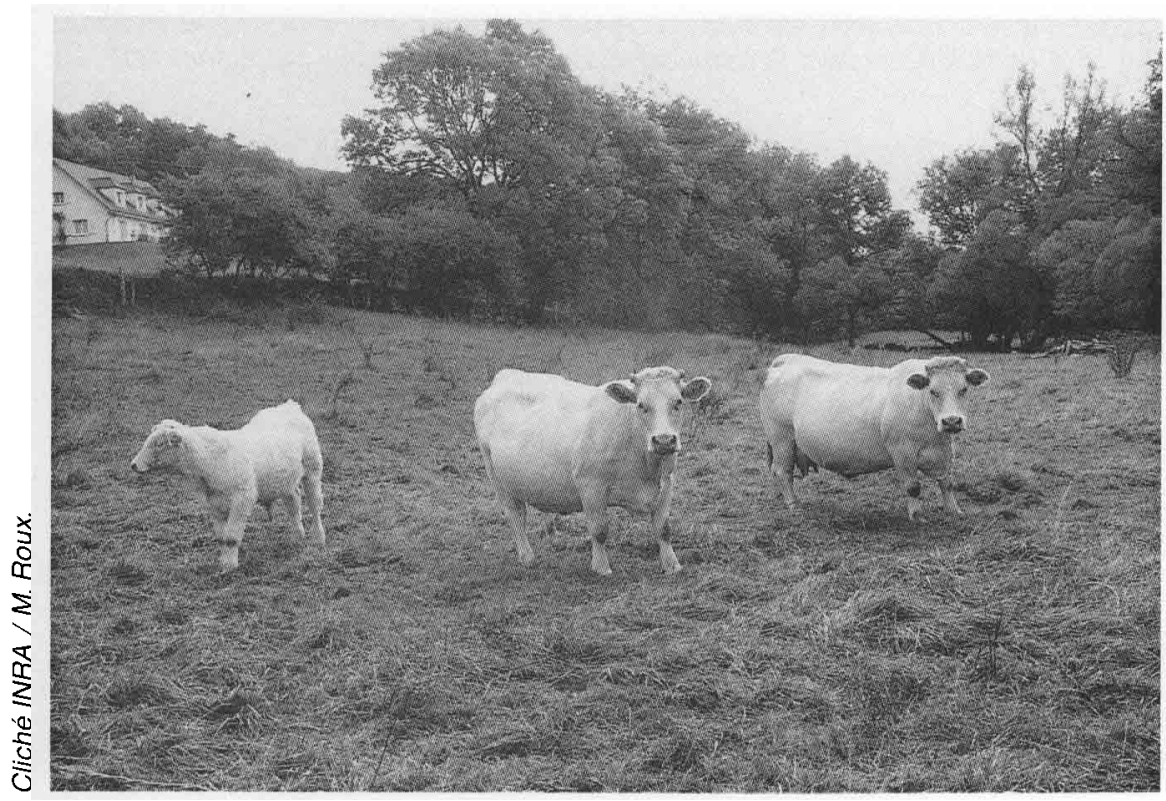

3 heures à $12^{\circ} \mathrm{C}$ environ puis refroidies en salle de réfrigération rapide $(+2 \% \mathrm{C}$ avec ventilation forcée) pendant 24 heures.

Des analyses sensorielles ont été effectuées pour tous les animaux ayant fait l'objet de dissection complète de la demi-carcasse. Ces analyses ont porté sur les muscles long dorsal (faux filet) et gros anconé (macreuse à bifteck) prélevés puis emballés sous vide et conservés 14 jours à $0^{\circ} \mathrm{C}$ afin d'obtenir une maturation complète. A ce stade, les muscles ont été coupés en tranches de $2 \mathrm{~cm}$ d'épaisseur, emballés sous vide puis congelés rapidement à $-20^{\circ} \mathrm{C}$ et conservés à cette température jusqu'au jour de l'analyse. Les steaks ont été décongelés pendant la nuit précédant la séance d'analyse sensorielle puis cuits sur un gril double face pendant 3 minutes environ afin d'atteindre une température à coeur de 55-60 $\mathrm{C}$. Les séances de dégustation ont eu lieu le matin entre $11 \mathrm{~h}$ et $12 \mathrm{~h}$ avec la participation à chaque séance de 10 personnes ayant suivi au préalable 10 séances d'entraînement. Elles ne connaissaient ni la provenance des échantillons, ni le protocole suivi. Pendant une séance d'une heure, quatre comparaisons ont été effectuées. Les échantillons à comparer (maigre - gras, jeune âgé, avec ou sans anabolisant) étaient présentés simultanément deux à deux. Les dégustateurs disposaient de pain et d'eau pendant le test et notaient sur une échelle à 10 points :

- la tendreté de 0 (très dur) à 10 (très tendre)

- la jutosité de 0 (très sec) à 10 (très juteux)

- l'intensité de la flaveur de $\mathbf{0}$ (très faible) à 10 (très intense)

Des mesures physico-chimiques ont été faites pour tous les animaux de l'essai 2 (40 au total) sur les muscles long dorsal et rhomboïde thoracique prélevés au niveau des $5^{e}$ et $6^{e}$ côtes d'une même demi-carcasse. Les mesures suivantes ont été faites sur viande fraîche ayant subi une maturation de 5 jours à $+4^{\circ} \mathrm{C}$ enveloppée dans une feuille d'aluminium :

La force de cisaillement avec l'appareil à cisailler INRA (Salé 1971)

Pour chaque muscle, 20 à 25 mesures ont été réalisées pour des épaisseurs variables de l'éprouvette de viande comprises entre 0,4 et $1,3 \mathrm{~cm}$. La force $(\mathrm{F})$ et le travail (W) de cisaillement ont été calculés par régression linéaire pour une épaisseur d'échantillon standard de $1 \mathrm{~cm}$. Ces mesures ont été faites sur viande crue et sur viande cuite. Pour la cuisson, des morceaux pesant $50 \mathrm{~g}$ environ ont été chauffés à l'étuve jusqu'à une température à coeur de $55-56^{\circ} \mathrm{C}$

- le pH sur viande broyée

- la teneur en matière sèche par dessiccation à l'étuve $\left(103^{\circ} \mathrm{C}\right.$ pendant 48 heures $)$ de 2 prises d'essai d'environ $10 \mathrm{~g}$ de muscle broyé au robot-coupe

- le pouvoir de rétention d'eau par mesure de la perte d'eau sous l'effet d'une pression selon la méthode de Goutefongea (1963)

- les pertes de poids à la cuisson mesurées au moment de la cuisson mentionnée ci-dessus

- la teneur en fer héminique selon la méthode de Hornsey (1956)

- la réflectance au moyen d'un réflectomètre Manuflex II.

Certaines analyses chimiques ont été effectuées sur viande congelée afin de connaître la teneur en collagène total (hydroxyproline x 7,5 ) selon une méthode adaptée de Bonnet et Kopp (1984), et la teneur en lipides totaux par extraction à froid avec un mélange chloroformeméthanol (rapport $2 / 1$ en volume) selon une méthode adaptée de Folch et al (1957).

\section{5 / Interprétation statistique des résultats}

Les résultats ont été interprétés par analyse de variance d'un dispositif factoriel non orthogonal, non équilibré, avec interaction dans lequel les facteurs suivants ont été pris en compte : 
- âge et anabolisant pour les performances d'engraissement de l'essai 2 ,

- état d'engraissement, âge et anabolisant pour les caractéristiques physico-chimiques musculaires de l'essai 2.

Les résultats des analyses sensorielles ont été analysés par le calcul des moyennes et l'analyse de variance, en tenant compte des effets muscle, facteur étudié et animal (logiciel SAS).

\section{2 / Résultats}

\section{1 / Performances d'engraissement des vaches}

\section{a / Poids, gain de poids et efficacité alimentaire}

Les reprises de poids à état d'engraissement optimum sont en moyenne très voisines dans les deux essais; le gain de poids vif se situe entre $1150 \mathrm{~g} / \mathrm{j}$ au cours des 70 premiers jours d'engraissement pour l'ensemble des animaux de l'essai 1 et $1130 \mathrm{~g} / \mathrm{j}$ sur 56 jours pour les animaux non implantés de l'essai 2 . La reprise de poids est cependant très variable d'un animal à l'autre, de 220 à $2130 \mathrm{~g} / \mathrm{j}$ pour les valeurs les plus extrêmes (coefficient de variation de 30 à $40 \%$ selon les lots).

Pour réaliser ces gains de poids, les vaches ont consommé en moyenne $13,7 \mathrm{~kg}$ de matière sèche totale par jour (tableau 3). Comparé au niveau de consommation de vaches Limousines engraissées dans les mêmes conditions et ayant des reprises de poids voisines (Malterre et al 1989), les vaches Charolaises ont ingéré environ $15 \%$ de plus, à même poids moyen et à même durée d'engraissement.

\section{- Effet de la durée d'engraissement (essai 1)}

La reprise de poids est très rapide au début de la période d'engraissement $(1330 \mathrm{~g} / \mathrm{j}$ en moyenne sur 6 semaines) puis diminue au delà du $50^{\mathrm{e}}$ jour (figure 2). De 70 à 109 jours elle n'est plus que de $650 \mathrm{~g} / \mathrm{j}$ environ. Ce ralentissement est semblable à celui observé chez des vaches laitières de race Normande en finition (Béranger et al 1970) ou chez des vaches allaitantes Hereford et Angus (Matulis et al 1987). Les quantités ingérées évoluent de façon similaire (figure 2). La consommation totale augmente rapidement de 12,5 à $14,5 \mathrm{~kg}$ de matière sèche par jour au cours des 35 premiers jours d'engraissement puis diminue légèrement jusqu'à $13 \mathrm{~kg}$ à partir de 70 jours.

L'indice de consommation cumulé augmente au cours de l'engraissement de $7,8 \mathrm{~kg}$ de matière sèche par $\mathrm{kg}$ de gain au début à $12,7 \mathrm{~kg}$ en fin d'engraissement (figure 3). Globalement l'efficacité alimentaire est peu modifiée par l'allongement de la durée d'engraissement jusqu'à 98 jours, environ $90 \mathrm{~g}$ de croît par UFL ingérée (tableau 3).

L'état d'engraissement estimé in vivo par maniements et par mesure du diamètre des adipocytes augmente au cours des 65 premiers jours d'engraissement et se poursuit au delà, jusqu'à 109 jours (tableau 3).
Figure 2. Evolution du poids vif des vaches et des quantités ingérées au cours de la période d'engraissement (essai 1).

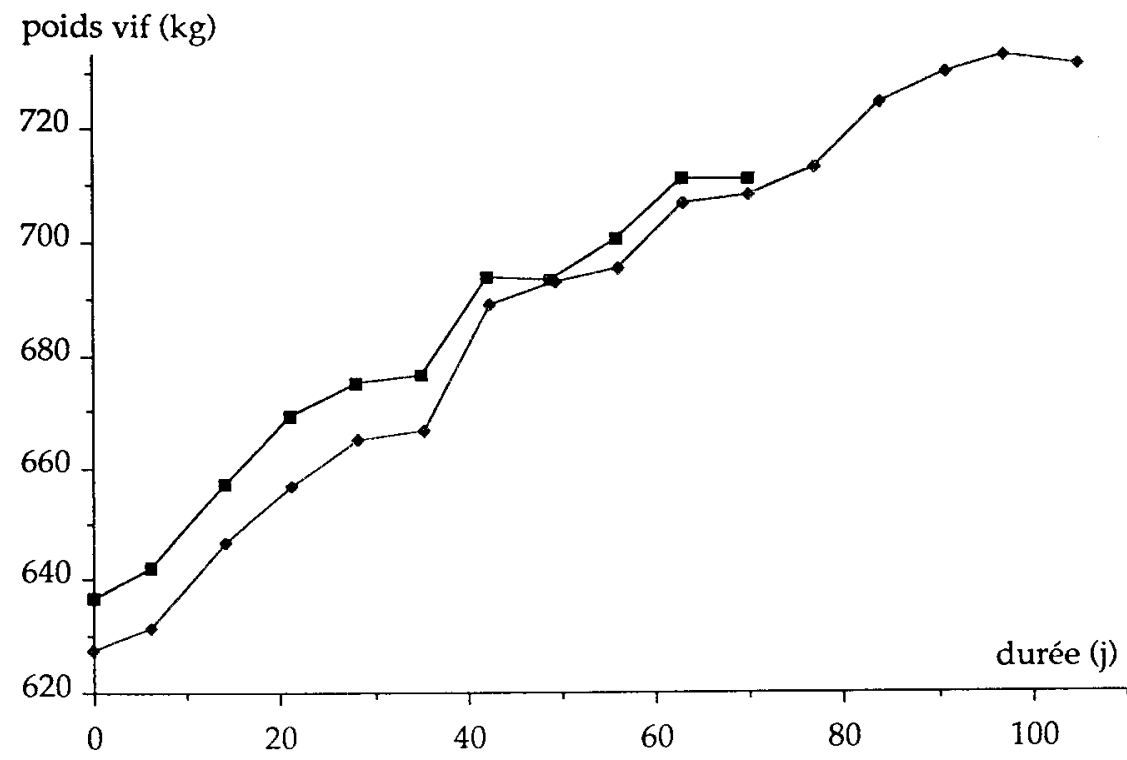

MS totale ingérée $(\mathrm{kg} / \mathrm{j})$

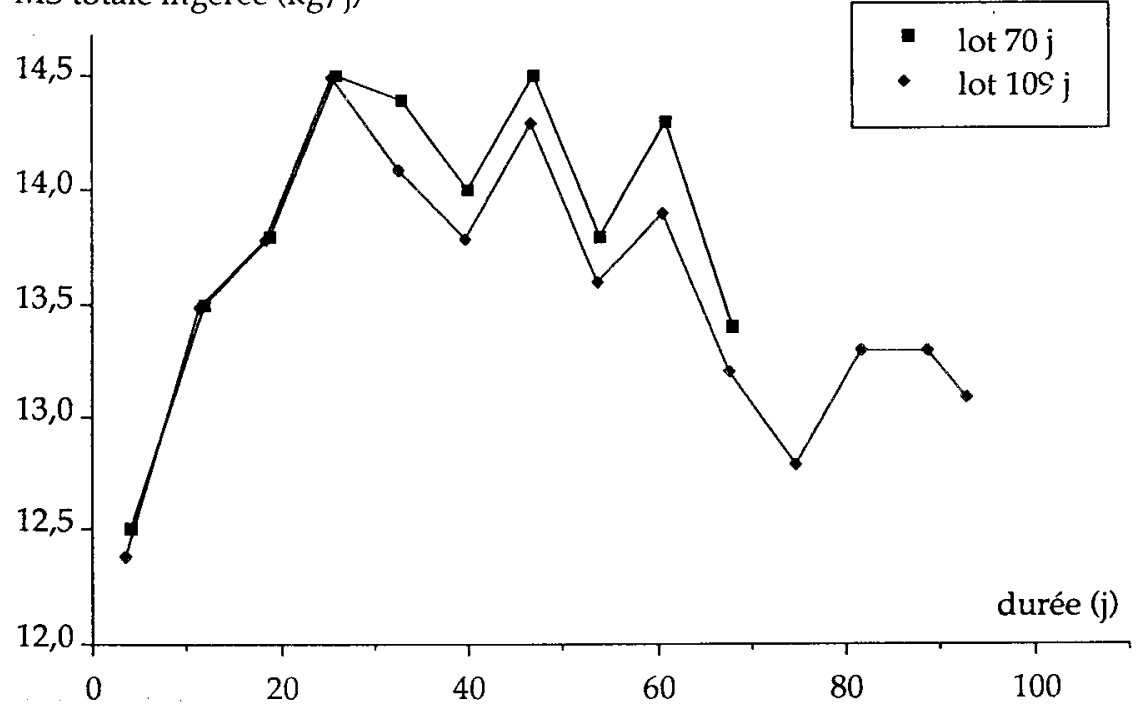

Figure 3. Evolution de l'indice de consommation cumulé ( $\mathrm{kg}$ MS ingérée/ $\mathrm{kg}$ gain de poids vif) au cours de la période d'engraissement (essai 1).

kg MS ingérée / kg gain PV

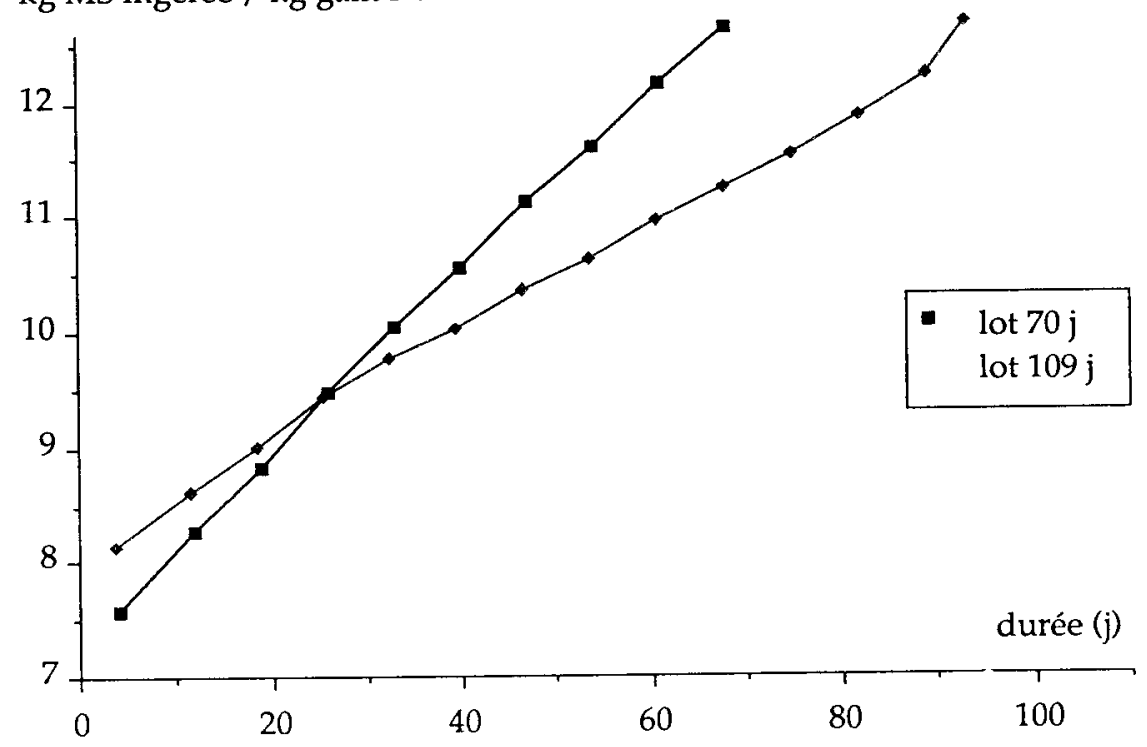


Tableau 3. Performances d'engraissement des vaches.

\begin{tabular}{|c|c|c|c|c|c|c|c|c|}
\hline \multirow[b]{2}{*}{ Lot } & \multicolumn{2}{|c|}{ Essai 1} & \multicolumn{4}{|c|}{ Essai 2} & \multicolumn{2}{|c|}{ source de variation } \\
\hline & G1 & $\mathrm{G} 2$ & JT & $\mathrm{AT}$ & JI & AI & âge & implant \\
\hline Nombre d'animaux & 13 & 10 & 9 & 6 & 9 & 9 & & \\
\hline Durée d'engraissement (j) & 70 & 109 & 56 & 56 & 83 & 83 & - & - \\
\hline $\begin{array}{l}\text { Poids vif initial }(\mathrm{kg}) \\
\text { (et) }\end{array}$ & $\begin{array}{l}635 \\
(64)\end{array}$ & $\begin{array}{l}626 \\
(59)\end{array}$ & $\begin{array}{l}600 \\
(70)\end{array}$ & $\begin{array}{l}665 \\
(71)\end{array}$ & $\begin{array}{l}619 \\
(42)\end{array}$ & $\begin{array}{l}672 \\
(79)\end{array}$ & * & ns \\
\hline $\begin{array}{l}\text { Poids vif final }(\mathrm{kg}) \\
\text { (et) }\end{array}$ & $\begin{array}{c}712 \\
(78)\end{array}$ & $\begin{array}{l}737 \\
(60)\end{array}$ & $\begin{array}{l}673 \\
(77)\end{array}$ & $\begin{array}{l}715 \\
(63)\end{array}$ & $\begin{array}{l}745 \\
(60)\end{array}$ & $\begin{array}{c}783 \\
(103)\end{array}$ & $n S^{(1)}$ & $* *(1\}$ \\
\hline $\begin{array}{l}\text { Gain de poids vif }(\mathrm{g} / \mathrm{j}) \\
\text { (et) }\end{array}$ & $\begin{array}{r}1100 \\
(380)\end{array}$ & $\begin{array}{l}1010 \\
(240)\end{array}$ & $\begin{array}{l}1300 \\
(480)\end{array}$ & $\begin{array}{c}890 \\
(390)\end{array}$ & $\begin{array}{r}1520 \\
(450)\end{array}$ & $\begin{array}{l}1340 \\
(460)\end{array}$ & $n s$ & * \\
\hline $\begin{array}{l}\text { Etat d'engraissement final } \\
\text { Note d'état }(/ 5) \\
\text { Diamètre des adipocytes }(\mu)\end{array}$ & $\begin{array}{l}2,7 \\
66\end{array}$ & $\begin{array}{l}3,7 \\
97\end{array}$ & 2,8 & 2,7 & 3,8 & 3,6 & $n s$ & ** \\
\hline $\begin{array}{l}\text { Quantités ingérées } \\
\mathrm{kg} \mathrm{MSI} / \mathrm{j}\end{array}$ & 13,9 & $13,6^{(2)}$ & 13,3 & 13,9 & 13,6 & 14,1 & - & - \\
\hline $\begin{array}{l}\text { kg MS/100 kg PV/j } \\
\text { Apports énergétiques }\end{array}$ & 2,19 & 2,17 & 2,22 & 2,10 & 2,19 & 2,10 & - & - \\
\hline $\begin{array}{l}\text { UFL/jour } \\
\text { UFL/kg de gain }\end{array}$ & $\begin{array}{l}12,1 \\
11,0\end{array}$ & $\begin{array}{l}11,8 \\
11,7\end{array}$ & $\begin{array}{l}12,4 \\
9,5\end{array}$ & $\begin{array}{l}13,0 \\
14,5\end{array}$ & $\begin{array}{l}12,6 \\
8,3\end{array}$ & $\begin{array}{c}13,2 \\
9,9\end{array}$ & $\begin{array}{l}- \\
-\end{array}$ & $\begin{array}{l}- \\
-\end{array}$ \\
\hline $\begin{array}{l}\text { Efficacité alimentaire } \\
\mathrm{kg} \text { MS/kg de gain } \\
\mathrm{g} \text { de croît/UFL }\end{array}$ & $\begin{array}{c}12,6 \\
91\end{array}$ & $\begin{array}{c}12,7 \\
90\end{array}$ & $\begin{array}{l}10,3 \\
105\end{array}$ & $\begin{array}{c}15,6 \\
69\end{array}$ & $\begin{array}{l}8,9 \\
120\end{array}$ & $\begin{array}{l}10,6 \\
101\end{array}$ & - & - \\
\hline
\end{tabular}

(1) avec introduction de la covariable « poids vif initial».

[2] Les mesures des quantités ingérées et d'efficacité alimentaire ont été faites sur 98 jours d'engraissement seulement.

(3) Poids vif début expérience.

(et) Ecart type

${ }^{*}$ significatif au seuil de $5 \%,{ }^{*}$ significatif au seuil de $1 \%$, ns non significatif.

\section{- Effet de l'âge (essai 2)}

Sur l'ensemble de la population (témoins et implantés confondus), les animaux jeunes ont un gain de poids vif supérieur de $250 \mathrm{~g}$ par jour à celui des animaux âgés (tableau 3). Cet écart n'est pas significatif mais il faut noter qu'il est beaucoup plus accentué entre les lots témoins (410 g) qu'entre les lots implantés (180 g). L'effectif du lot âgé témoin $(n=6)$ est trop faible pour conclure à un effet net de l'âge sur la reprise de poids vif dans le cadre de cet essai, d'autant plus que les évolutions du poids du contenu digestif diffèrent entre les lots jeunes et âgés au cours de l'engraissement. Ainsi chez les animaux témoins disséqués, l'écart de $26 \mathrm{~kg}$ de contenu digestif observé entre âgés et jeunes chez les animaux maigres abattus en début d'engraissement ne se retrouve pas après un engraissement de 56 jours (tableau 6).

Pour l'ensemble des animaux, les quantités ingérées sont élevées dès le début de l'engraissement (en moyenne $13,8 \mathrm{~kg}$ de matière sèche par jour) et atteignent $14,8 \mathrm{~kg}$ de matière sèche vers le $45^{\mathrm{e}}$ jour (figure 4 ).

Pesant $65 \mathrm{~kg}$ de moins au début de l'essai, les jeunes vaches du lot témoin (JT) ingèrent aussi $0,6 \mathrm{~kg}$ de matière sèche de moins par jour. Ainsi leur indice de consommation global est inférieur de 5,3 points à celui de leurs homologues âgées (tableau 3). Ces résultats confirment les observations de Malterre et al (1989) sur des vaches Limousines: au-delà de 11 ans, le niveau d'ingestion diminue, mais proportionnellement toujours moins que les performances de croissance. Ainsi l'efficacité alimentaire diminue toujours avec l'âge. Cependant cette diminution des performances liée à l'âge s'observe surtout chez des animaux très âgés; Jones et Macleod (1981) comparant des vaches Holstein jeunes à des adultes pas très âgées ne trouvent aucune différence significative de reprise de poids vif et d'indice de consommation au bout de 68 jours d'engraissement.

A l'issue d'une durée d'engraissement de 56 jours, et malgré une vitesse de reprise de poids beaucoup plus rapide chez les jeunes vaches, l'état d'engraissement estimé in vivo est pratiquement identique pour les animaux témoins (lots JT et AT, tableau 3).

\section{- Effet d'un anabolisant (essai 2)}

Les vaches ayant reçu un implant d'acétate de trenbolone, quel que soit leur âge, reprennent en moyenne $22 \mathrm{~kg}$ de poids vif de plus que les témoins ( $1530 \mathrm{~g} / \mathrm{j}$ contre $1135 \mathrm{~g} / \mathrm{j}, \mathrm{P}<$ 0,05 ) au cours des 56 premiers jours d'engraissement. La poursuite de l'engraissement jusqu'au $83^{\circ}$ jour n'entraîne pratiquement pas de ralentissement de la vitesse de croissance (figure 4).

Ainsi chez les animaux implantés, le gain de poids journalier calculé sur toute la période d'engraissement (83 jours) reste supérieur $(\mathrm{P}<$ 0,05 ) à celui des animaux témoins réalisé en 56 jours (tableau 3). L'effet positif de l'anabolisant sur le gain de poids paraît donc bien se prolonger et se conserver au delà du délai préconisé de 60 jours après administration. 
L'implantation n'a pas d'effet marqué sur le niveau d'ingestion des vaches (tableau 3) si bien que l'indice de consommation moyen des lots traités est amélioré de $22 \%$ par rapport à celui des témoins $(9,7 \mathrm{~kg}$ matière sèche par $\mathrm{kg}$ de gain contre 12,4). Cette amélioration de l'efficacité alimentaire est du même ordre que celle citée par Geav (1986): + $15 \%$ en moyenne chez les femelles.

\section{b / Résultats d'abattage}

\section{- Poids de carcasse et rendement à l'abattage}

Sans anabolisant, le poids moyen des carcasses chaudes se situe entre 380 et $400 \mathrm{~kg}$ (tableau 4). Après 70 jours d'engraissement l'état des carcasses est satisfaisant pour la plupart des marchés français : les dépôts adipeux de la carcasse se situent aux alentours de $18 \%$ du poids de la carcasse chaude (essai 1). Le rendement commercial (poids de carcasse froide/poids vif à l'abattage) est compris entre 53 et $55 \%$, inférieur de 3 points en moyenne à celui obtenu sur vaches Limousines (Malterre et al 1989). Cette différence s'explique en partie par un poids de contenu digestif plus élevé pour les Charolaises (15\% du poids vif contre $12 \%$ pour les Limousines). En terme de rendement vrai (poids de carcasse chaude/poids vif vide), l'écart est moins élevé (1,7 point).

L'allongement de la durée d'engraissement de 39 jours dans l'essai 1 se traduit par une augmentation du poids vif vide de $25 \mathrm{~kg}$ qui se répartissent en $19 \mathrm{~kg}$ de carcasse et $6 \mathrm{~kg}$ de $5^{\circ}$ quartier. Cette augmentation correspond essentiellement à des dépôts adipeux (tableau 4).

Entre les vaches jeunes (JT) et âgées (AT) l'écart de poids de carcasse chaude n'est que de $14 \mathrm{~kg}$ pour un écart de poids vif initial de 65 $\mathrm{kg}$ : les jeunes témoins compensent donc leur poids plus réduit par une meilleure croissance et par un rendement à l'abattage légèrement plus élevé $(+1,4$ point $)$. Cette différence peut être reliée au poids plus élevé $(\mathrm{P}<0,05) \mathrm{du}$ tube digestif vide et plus particulièrement de la caillette et des intestins pour les animaux âgés (tableau 4). Cette augmentation du poids du tractus digestif avec l'âge a déjà été observée chez des vaches Holstein (Smith et Baldwin 1974 ; Jones 1981). Il est cependant difficile d'expliquer l'augmentation du poids de la caillette avec l'âge. Jones (1981) a constaté un développement de cet organe chez des vaches engraissées avec de l'ensilage de maïs, par rapport à des vaches maigres, mais n'a pas observé de variation liée à l'âge des animaux.

Le facteur de croissance permet d'obtenir des poids de carcasse chaude plus élevés $(421 \mathrm{~kg}$ contre $385 \mathrm{~kg}$ chez les témoins, $\mathrm{P}<0,05$ ). Les poids du contenu digestif et de l'ensemble du tractus digestif sont plus élevés chez les vaches implantées (respectivement $\mathrm{P}<0,05$ et $\mathrm{P}<$ $0,01)$ sans incidence significative sur les rendements à l'abattage (tableau 4). Ces augmentations peuvent s'expliquer en partie par un niveau de consommation élevé jusqu'à 83 jours d'engraissement (figure 4). Une augmentation du poids total du tube digestif a déjà été constatée par Doreau et al (1985) sur des vaches laitières taries engraissées, mais ces auteurs constataient également que cette différence significative par rapport à des vaches maigres disparaît lorsqu'on compare les poids du tube
Figure 4. Evolution du poids vif des vaches et des quantités ingérées au cours de la période d'engraissement (essai 2).

poids vif $(\mathrm{kg})$

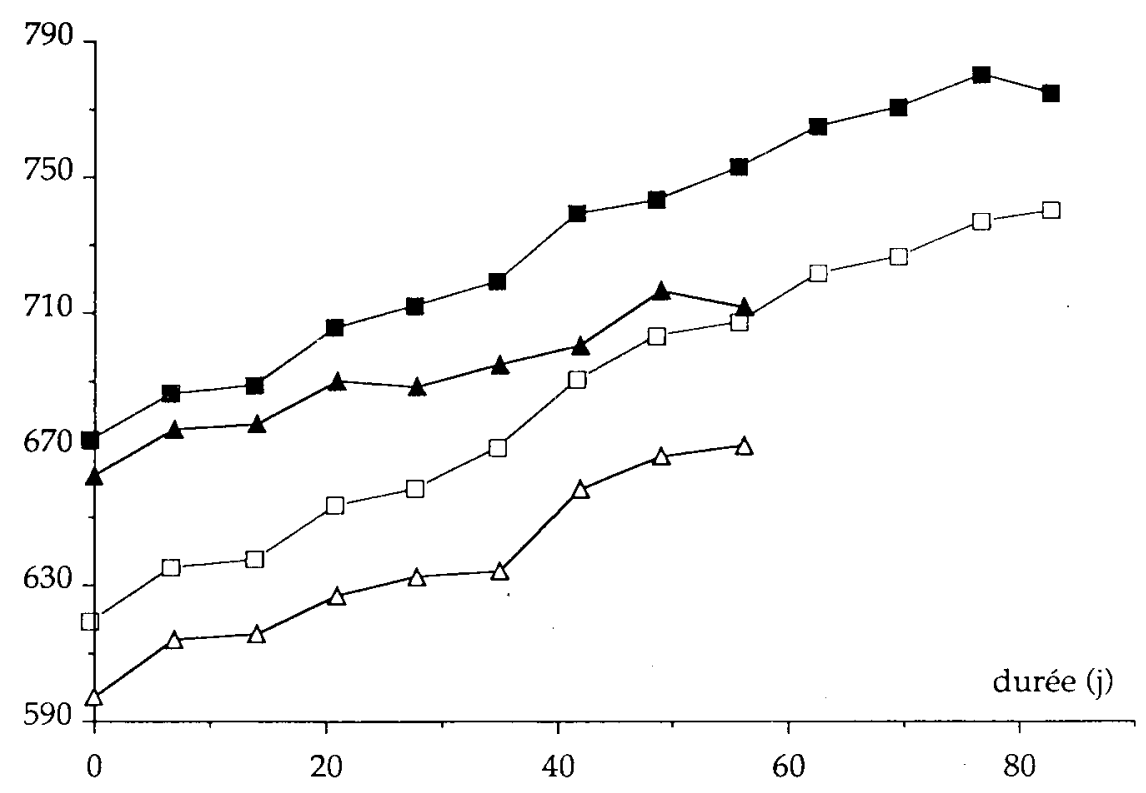

MS totale ingérée $(\mathrm{kg} / \mathrm{j})$

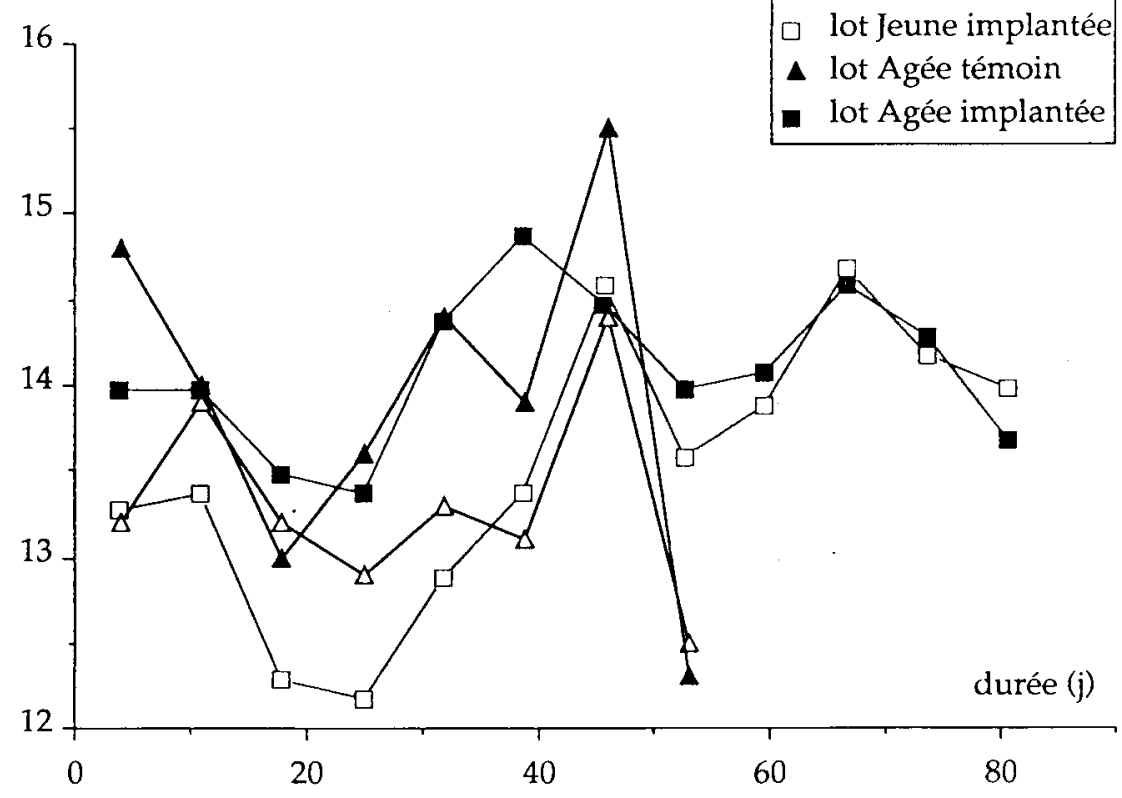

digestif délipidé. Or, il est clair que les animaux anabolisés sont plus gras à l'abattage, comme l'indique le développement important des dépôts adipeux du $5^{11}$ quartier $(+40 \%$ par rapport aux témoins, $\mathrm{P}<0,01$ ) (tableau 4).

\section{- Composition tissulaire des carcasses}

L'allongement de 39 jours de la durée d'engraissement se traduit par une plus forte proportion de dépôts adipeux dans la carcasse $(+4$ points). Le poids moyen de muscle de la carcasse est sensiblement le même pour les deux durées d'engraissement (70 et 109 jours); la différence de poids de carcasse chaude correspond essentiellement à l'écart de poids des dépôts adipeux (tableau 4 et figure 5). 
Tableau 4. Résultats d'abattage et estimation de la composition de la carcasse (dissection $6^{e}$ côte).

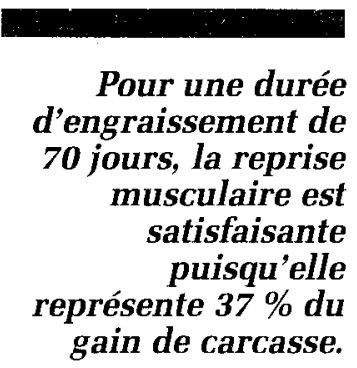

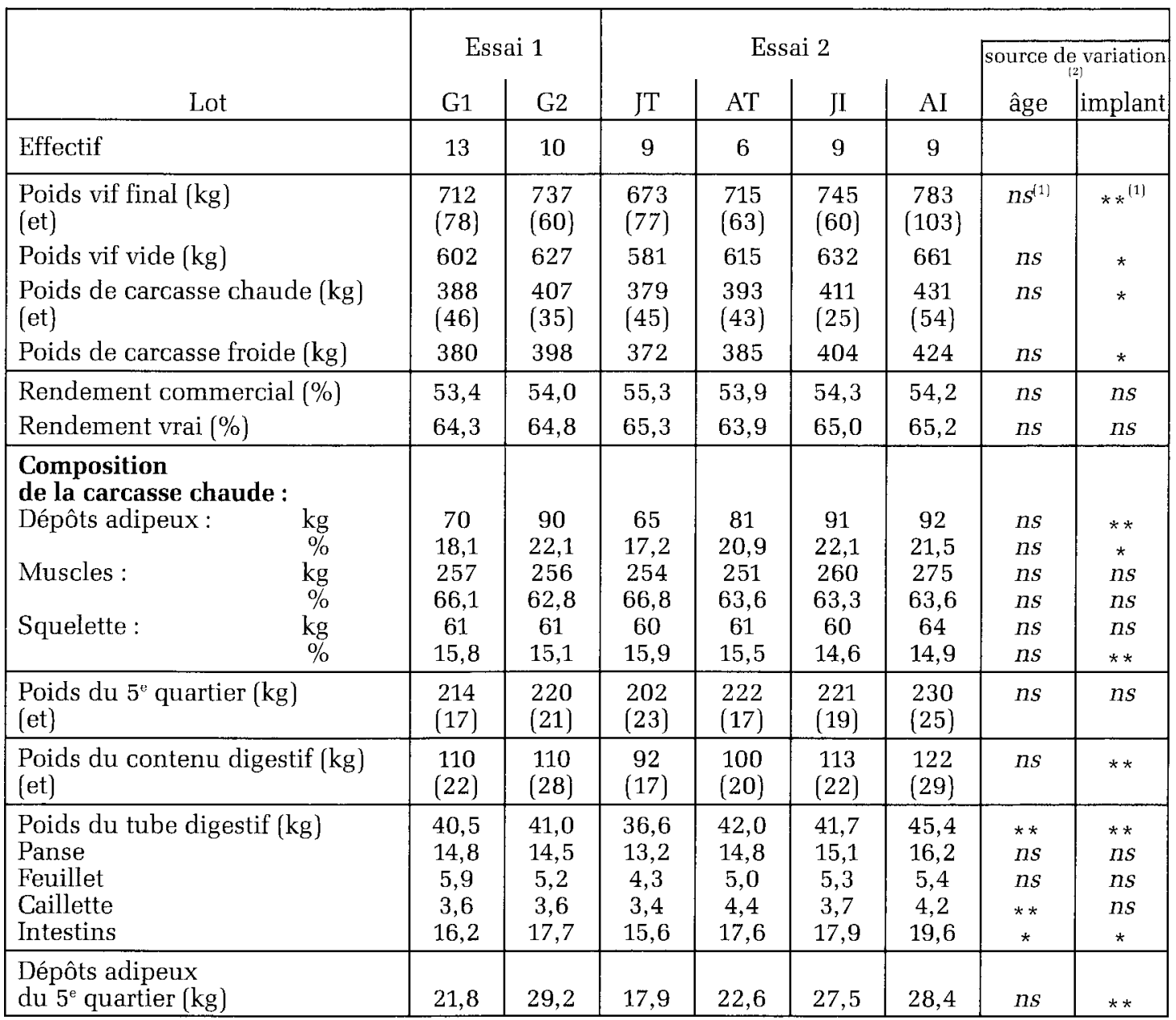

(1) avec correction pour les variations de poids initial introduit comme covariable dans l'analyse de variance.

${ }^{(2)}$ * significatif au seuil de $5 \%, * *$ significatif au seuil de $1 \%, n s$ non significatif.

(et) Ecart type.

Tableau 5. Mensurations des carcasses de l'essai 2.

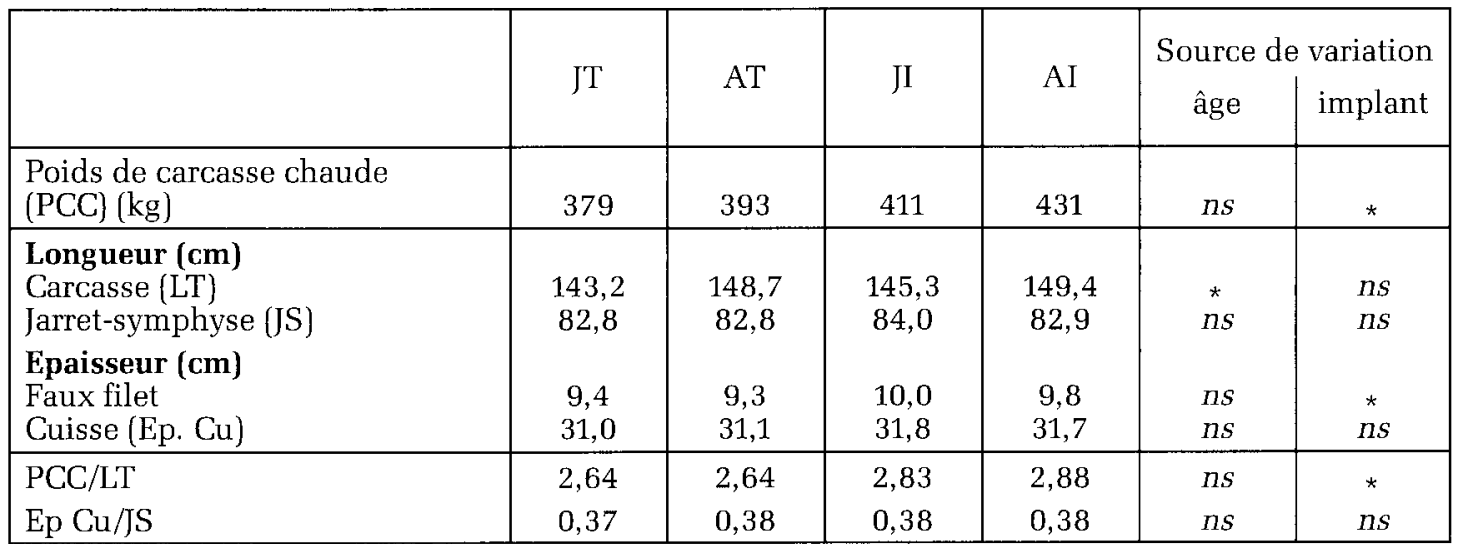

* significatif au seuil de $5 \%, * *$ significatif au seuil de $1 \%$, ns non significatif.

Pour l'ensemble des animaux de l'essai 2 (témoins et implantés), l'âge n'a pas d'effet significatif sur la composition tissulaire des carcasses (tableau 4). Chez les animaux témoins $(n=15)$, les carcasses sont en moyenne constituées de $18,7 \%$ de tissu adipeux, $65,5 \%$ de tissu musculaire et $15,7 \%$ de squelette: valeurs très proches de celles obte- nues dans l'essai 1 pour le lot engraissé 70 jours (G1).

Du fait de l'allongement excessif de la durée d'engraissement, l'état d'adiposité final des carcasses est supérieur dans les lots implantés contrairement à l'objectif visé : les dépôts adipeux rapportés au poids de carcasse chaude 
sont respectivement de $21,8 \%$ et de $18,7 \%$ pour les lots implantés et témoins $(\mathrm{P}<0,05)$ (tableau 4). Cet écart est surtout marqué chez les vaches jeunes.

\section{- Conformation des carcasses}

L'utilisation d'un anabolisant permet d'améliorer la conformation des carcasses appréciée par mensurations (tableau 5), notamment l'épaisseur de faux filet et la compacité (poids de carcasse chaude/longueur totale).

\section{c / Composition du gain de carcasse et bilan énergétique}

\section{- Composition du gain de carcasse}

Le gain de carcasse et sa composition ont été déterminés en comparant les résultats de dissection d'animaux maigres en début d'expérience et d'animaux engraissés (tableau 6). Ainsi pour une durée d'engraissement de 109 jours, la reprise de poids vif vide est de $131 \mathrm{~kg}$ pour les lots d'animaux homologues disséqués, soit un gain de poids de carcasse de $92 \mathrm{~kg}$ qui se décompose en $26 \mathrm{~kg}$ de muscle et $66 \mathrm{~kg}$ de tissu adipeux, respectivement $29 \%$ et $71 \%$ du gain de carcasse (figure 5). Ce résultat est comparable à la composition du gain de carcasse observé sur des vaches Limousines engraissées 123 jours (Malterre et al 1989) et dans d'autres conditions sur des vaches Hereford (Wooten et al 1979). Il souligne la capacité de reprise musculaire des vaches de réforme de race à viande par rapport aux races laitières (Chilliard et al 1984). Toutefois, ce gain de muscle ne correspond pas à un accroissement préférentiel de muscles à valeur bouchère élevée (Robelin et al 1990) ; ces résultats confirment donc dans leur ensemble les observations de Malterre et al (1989) sur vaches Limousines.

La reprise de poids musculaire se fait essentiellement en début d'engraissement. Les estimations de la composition des carcasses (dissection de la $6^{\circ}$ côte) des animaux engraissés durant 70 jours indiquent que la reprise de poids de carcasse de $64 \mathrm{~kg}$ comprend environ $23 \mathrm{~kg}$ de muscle ce qui correspond à la quasitotalité de la reprise musculaire en 109 jours d'engraissement. Ainsi, l'accroissement du poids de carcasse au-delà de 70 jours correspond essentiellement à des dépôts adipeux. Ces résultats sont en accord avec les estimations réalisées sur l'ensemble de la population (tableau 4) qui mettent en évidence que les animaux produisent approximativement le même poids de muscle $(256 \mathrm{~kg}$ ) en 70 ou 109 jours d'engraissement. D'après nos estimations, le gain de carcasse obtenu au cours d'un engraissement d'une durée de 70 jours est composé approximativement de $37 \%$ de tissu musculaire et de $63 \%$ de tissu adipeux (figure 5 ).

Dans l'essai 2, le gain de tissu musculaire au cours de l'engraissement, estimé à partir des dissections, est important : de 37 à $38 \mathrm{~kg}$ soit environ $40 \%$ du gain de carcasse. Cette valeur, plus élevée que celle obtenue dans l'essai 1 , est vraisemblablement très dépendante du choix de la date d'abattage des vaches maigres; or il existait des différences sur ce point entre les 2 essais (figure 1). Cette reprise musculaire est indépendante de l'âge des animaux. Ce résultat pouvait être attendu puisque les carcasses des 2
Figure 5.Composition des carcasses et du gain de carcasse des lots homologues disséqués.

\section{Effet de la durée d'engraissement}

Composition de la carcasse Composition du gain de carcasse

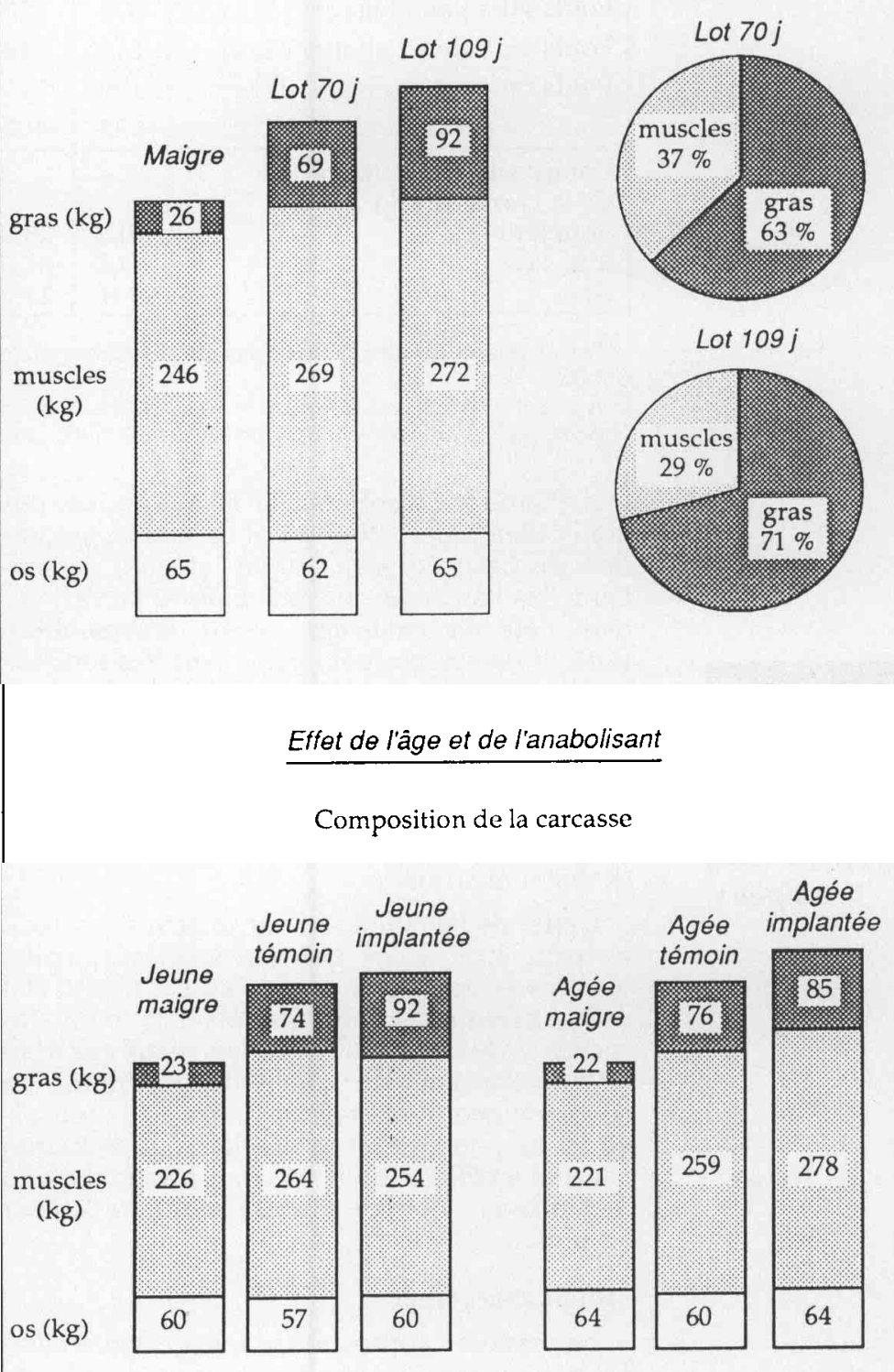

Composition du gain de carcasse
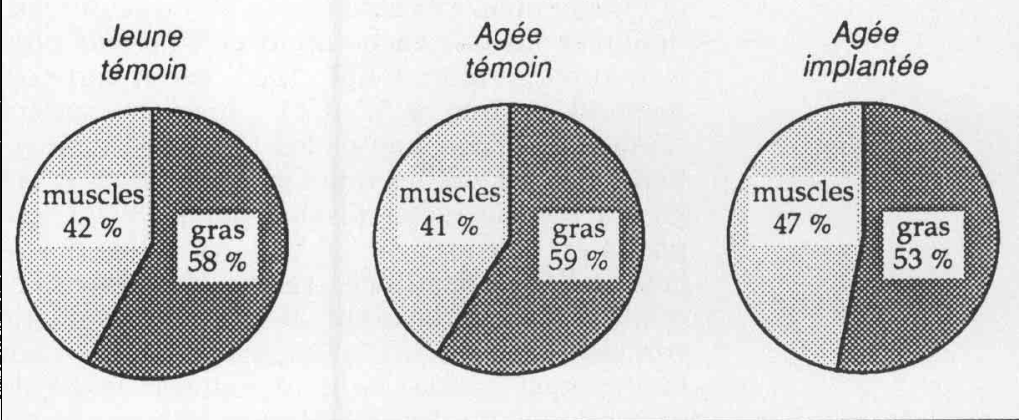
Tableau 6. Résultats d'abattage et composition de la carcasse des vaches des lots homologues disséqués.

\begin{tabular}{|l|c|c|c|c|c|c|c|c|c|}
\hline & \multicolumn{3}{c|}{ Essai 1 } & \multicolumn{5}{c|}{ Essai 2 } \\
\multicolumn{1}{|c|}{ Lot } & M & G1 & G2 & JM & JT & JI & AM & AT & AI \\
\hline Nombre d'animaux & 4 & 5 & 5 & 3 & 4 & 4 & 4 & 4 & 4 \\
Poids vif sur régime foin & & & & & & & & & \\
(début transition) kg & - & - & - & 598 & 596 & 601 & 637 & 637 & 637 \\
Poids vif à l'abattage (kg) & 621 & 738 & 773 & 598 & 686 & 746 & 637 & 704 & 780 \\
Poids vif vide à l'abattage (kg) & 512 & 616 & 643 & 466 & 596 & 623 & 479 & 614 & 651 \\
Poids du contenu digestif (kg) & 109 & 122 & 130 & 132 & 90 & 123 & 158 & 90 & 129 \\
Poids de carcasse chaude (kg) & 337 & 400 & 429 & 309 & 395 & 406 & 307 & 395 & 427 \\
\hline Composition tissulaire & & & & & & & & & \\
de la carcasse (\%) ${ }^{(3)}$ & & & & & & & & & \\
Squelette & 19,2 & 15,5 & 15,2 & 19,3 & 14,5 & 14,9 & 20,9 & 15,3 & 15,0 \\
Muscles & 73,0 & 67,2 & 63,4 & 73,4 & 66,7 & 62,5 & 72,0 & 65,6 & 65,2 \\
Gras & 7,8 & 17,2 & 21,4 & 7,3 & 18,8 & 22,6 & 7,1 & 19,1 & 19,8 \\
\hline
\end{tabular}

(1) Les animaux du lot G1 n’ont pas été disséqués ; la composition de la carcasse est estimée par dissection de la $6^{\varepsilon}$ côte.

(2) Après correction pour tenir compte des écarts de poids vif entre les lots à la mise en lot.

(3) Après élimination de la proportion de déchets, comprise entre 0,8 et $0,4 \%$ du poids de carcasse.

lots d'animaux disséqués (JT et AT) avaient des poids identiques $(395 \mathrm{~kg}$ ) et la même proportion de tissu adipeux (19\%), et que, par ailleurs, les carcasses des lots maigres correspondants étaient également assez comparables. Dans la musculature totale, la part des muscles à valeur bouchère élevée (muscles du dos et de la cuisse) est légèrement plus élevée chez les vaches jeunes $(51,2 \%)$ que chez le vaches âgées $(50,2 \%)$. Cette proportion se conserve après engraissement chez les jeunes $(51,0 \%)$, mais tendrait à diminuer chez les âgées $(48,4 \%)$ (Robelin et al 1990).

L'effet de l'anabolisant sur le gain de muscle ne peut être évalué que lorsque les animaux témoins et implantés sont abattus à même état d'engraissement donc uniquement pour les vaches âgées (tableau 6). Ainsi pour ces dernières, l'acétate de trenbolone entraîne un accroissement de la reprise de poids de muscle de $19 \mathrm{~kg}$ ' pour les lots disséqués qui se traduit par une amélioration de la composition du gain de carcasse : $48 \%$ de muscle contre $41 \%$ pour les témoins (figure 5).

\section{- Bilan énergétique}

En première approximation, le besoin d'énergie nette nécessaire à la constitution d'un $\mathrm{kg}$ de masse corporelle peut être calculé à partir des apports énergétiques totaux diminués des besoins d'entretien. En prenant comme base de calcul des dépenses d'entretien de $105 \mathrm{kcal}$ d'énergie métabolisable par $\mathrm{kg} \mathrm{P}^{0.75}$ (recommandations pour une vache nourrice à activité physiologique réduite, INRA, 1988) cet apport correspond environ à 5,2 UFL; mais en partant d'une hypothèse haute des dépenses d'entretien pour des vaches qui s'engraissent, soit 140

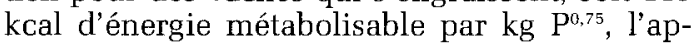
port n'est plus que de 3,8 UFL par kg de masse corporelle reconstituée, valeur correspondant mieux à la composition observée du gain de carcasse. Calculé sur les mêmes bases, le coût énergétique nécessaire pour réaliser un $\mathrm{kg}$ de gain augmente fortement avec l'âge: il est supérieur de $50 \%$ environ chez les vaches âgées par rapport aux jeunes qui se situent entre 2,5 et 3,0 UFL dans cette expérience. Cependant la composition du gain de poids ne peut pas expliquer cet important écart qui doit provenir de la diminution globale des rendements d'utilisation de l'énergie métabolisable avec l'âge.

\section{2 / Caractéristiques organoleptiques et physico-chimiques des viandes}

\section{a / Caractéristiques organoleptiques (tableau 7)}

L'effet de l'état d'engraissement des carcasses sur les qualités organoleptiques de la viande a été testé dans les 2 essais. Dans l'essai 1 , les animaux maigres $(7,8 \%$ de tissu adipeux dans la carcasse) âgés de 8,5 ans étaient comparés aux animaux engraissés 109 jours (21,4\% de tissu adipeux) âgés de 8,4 ans. Dans l'essai 2 , le lot maigre $(7,2 \%$ de tissu adipeux) âgé de 8,8 ans en moyenne était comparé au lot engraissé 56 jours sans anabolisant $(19,1 \%$ de tissu adipeux) et au lot engraissé 83 jours avec anabolisant $(20,9 \%$ de tissu adipeux $)$ âgés respectivement de 8,2 et 8,7 ans.

L'effet de l'état d'engraissement des carcasses sur les résultats de l'analyse sensorielle est remarquable, puisque les deux muscles étudiés (long dorsal et gros anconé) sont toujours jugés significativement plus tendres, de meilleure jutosité et de flaveur plus intense lorsque les carcasses sont grasses.

L'effet de l'âge est significatif pour la tendreté ; la viande des animaux jeunes (5 ans) est significativement plus tendre que celle des animaux âgés (11 ans): note moyenne de 5,45 contre 4,99. En revanche, aucun écart significatif dû à l'âge n'apparaît pour la jutosité ou la flaveur.

Enfin, l'administration d'un anabolisant n'a aucun effet significatif sur les notes moyennes de tendreté, de jutosité ou de flaveur. 


\begin{tabular}{|c|c|c|c|c|c|c|c|}
\hline & \multirow{2}{*}{ Effectif } & \multicolumn{2}{|c|}{ Tendreté } & \multicolumn{2}{|c|}{ Jutosité } & \multicolumn{2}{|c|}{ Flaveur } \\
\hline & & LD & GA & LD & GA & LD & GA \\
\hline \multicolumn{8}{|l|}{ Essai 1} \\
\hline Maigre (M) & 4 & 4,13 & 4,61 & 3,86 & 4,94 & 5,01 & 5,02 \\
\hline 109 j d'engrais ( $G 2$ ) & 5 & 6,23 & 5,08 & 5,56 & 5,50 & 5,36 & 5,82 \\
\hline $\begin{array}{l}\text { Seuil de signification } \\
\text { comparaison }(\mathrm{M})-(\mathrm{G} 2)\end{array}$ & & \multicolumn{2}{|c|}{$M \stackrel{*}{*} \mathrm{G} 2$} & \multicolumn{2}{|c|}{$M * *$} & \multicolumn{2}{|c|}{$M * *$} \\
\hline \multicolumn{8}{|l|}{$\begin{array}{l}\text { Essai } 2 \\
\text { Age } \times \text { engraissement }\end{array}$} \\
\hline \multicolumn{8}{|l|}{ Jeunes : $(J)$} \\
\hline maigre $(\mathrm{M})$ & 3 & 5,24 & 3,81 & 5,11 & 5,61 & 5,30 & 4,91 \\
\hline témoin (T) & 4 & 5,85 & 5,58 & 5,27 & 5,99 & 5,34 & 5,85 \\
\hline implanté (I) & 4 & 6,56 & 5,56 & 5,80 & 6,06 & 5,64 & 5,78 \\
\hline \multicolumn{8}{|l|}{ Agées: (A) } \\
\hline maigre (M) & 4 & 4,31 & 3,94 & 5,08 & 5,07 & 4,86 & 5,18 \\
\hline témoin $(\mathrm{T})$ & 4 & 5,30 & 5,74 & 5,08 & 6,10 & 5,14 & 5,72 \\
\hline implanté (I) & 4 & 5,44 & 5,20 & 5,16 & 5,87 & 5,32 & 5,72 \\
\hline \multicolumn{8}{|l|}{ Source de variation } \\
\hline Age : & & \multicolumn{2}{|c|}{$\mathrm{J}>\mathrm{*}$} & \multicolumn{2}{|c|}{$n s$} & \multicolumn{2}{|c|}{ ns } \\
\hline Engraissement & & \multicolumn{2}{|c|}{$M{ }^{*} \mathrm{~T}=\mathrm{I}$} & \multicolumn{2}{|c|}{$M \stackrel{*}{*}=I$} & \multicolumn{2}{|c|}{$\mathrm{M}<\stackrel{*}{\mathrm{~T}}=\mathrm{I}$} \\
\hline
\end{tabular}

* significatif au seuil de $5 \%, *$ significatif au seuil de $1 \%$, ns non significatif.

\begin{tabular}{|c|c|c|c|c|c|c|c|}
\hline & Effectif & M & $\begin{array}{l}\text { cle long d } \\
\text { Matière } \\
\text { sèche } \\
(\%)\end{array}$ & $\begin{array}{l}\text { rsal } \\
\text { Lipides } \\
\text { totaux } \\
\text { (\% frais) }\end{array}$ & Muscle & $\begin{array}{l}\text { Matière } \\
\text { sèche } \\
(\%)\end{array}$ & $\begin{array}{l}\text { horacique } \\
\text { Lipides } \\
\text { totaux } \\
\text { (\% frais) }\end{array}$ \\
\hline Moyenne générale & 40 & 5,52 & 26,09 & 3,73 & 5,55 & 25,53 & 4,35 \\
\hline $\begin{array}{l}\text { Age } \\
5 \text { ans } \\
11 \text { ans }\end{array}$ & $\begin{array}{l}21 \\
19\end{array}$ & $\begin{array}{c}n s \\
5,50 \\
5,54\end{array}$ & $\begin{array}{c}n s \\
26,05 \\
26,14\end{array}$ & $\begin{array}{c}n s \\
3,64 \\
3,83\end{array}$ & $\begin{array}{c}{ }^{* *} \\
5,51^{\mathrm{b}} \\
\mathbf{5}, 58^{\mathrm{a}}\end{array}$ & $\begin{array}{c}n s \\
25,74 \\
25,30\end{array}$ & $\begin{array}{c}n s \\
4,56 \\
4,13\end{array}$ \\
\hline $\begin{array}{l}\text { Engraissement } \\
\text { - maigre } \\
\text { - sans anabolisant } \\
\text { - avec anabolisant }\end{array}$ & $\begin{array}{r}7 \\
15 \\
18\end{array}$ & $\begin{array}{l}{ }^{* *} \\
5,66^{\mathrm{a}} \\
5,51^{b} \\
5,48^{\mathrm{b}}\end{array}$ & $\begin{array}{l}{ }^{* *} \\
23,94^{\mathrm{c}} \\
25,91^{\mathrm{b}} \\
27,08^{\mathrm{a}}\end{array}$ & $\begin{array}{l}{ }^{* *} \\
1,71^{\mathrm{c}} \\
3,78^{\mathrm{b}} \\
4,48^{\mathrm{a}}\end{array}$ & $\begin{array}{l}{ }^{* *} \\
5,71^{\text {a }} \\
5,52^{\mathrm{b}} \\
5,50^{\mathrm{b}}\end{array}$ & $\begin{array}{l}* * \\
24,16^{*} \\
25,33^{\mathrm{b}} \\
26,24^{\mathrm{a}}\end{array}$ & $\begin{array}{l}{ }^{* *} \\
2,47^{\mathrm{b}} \\
4,41^{\mathrm{a}} \\
5,04^{\mathrm{a}}\end{array}$ \\
\hline $\begin{array}{l}\text { Age } \times \text { engraissement } \\
5 \text { ans : }\end{array}$ & & $n s$ & $n s$ & $n s$ & * & $n s$ & $n s$ \\
\hline $\begin{array}{l}\text { - maigre } \\
\text { - sans anabolisant } \\
\text { - avec anabolisant }\end{array}$ & $\begin{array}{l}3 \\
9 \\
9\end{array}$ & $\begin{array}{l}5,60 \\
5,50 \\
5,48\end{array}$ & $\begin{array}{l}23,92 \\
25,65 \\
27,16\end{array}$ & $\begin{array}{l}1,56 \\
3,56 \\
4,42\end{array}$ & $\begin{array}{l}5,55 \\
5,51 \\
5,51\end{array}$ & $\begin{array}{l}23,71 \\
25,42 \\
26,74\end{array}$ & $\begin{array}{l}1,87 \\
4,47 \\
5,54\end{array}$ \\
\hline $\begin{array}{l}11 \text { ans : } \\
\text { - maigre } \\
\text { - sans anabolisant } \\
\text { - avec anabolisant }\end{array}$ & $\begin{array}{l}4 \\
6 \\
9\end{array}$ & $\begin{array}{l}5,71 \\
5,52 \\
5,48\end{array}$ & $\begin{array}{l}23,95 \\
26,31 \\
27,00\end{array}$ & $\begin{array}{l}1,82 \\
4,11 \\
4,53\end{array}$ & $\begin{array}{l}5,83 \\
5,54 \\
5,50\end{array}$ & $\begin{array}{l}24,51 \\
25,19 \\
25,74\end{array}$ & $\begin{array}{l}2,93 \\
4,32 \\
4,53\end{array}$ \\
\hline
\end{tabular}

* significatif au seuil de $5 \%, *$ significatif au seuil de $1 \%, n s$ non significatif

$a, b, c$ : les valeurs suivies de lettres différentes sont significativement différentes.

\section{b / Caractéristiques physico-chimiques des muscles (essai 2)}

\section{- Evolution post mortem}

L'évolution post mortem des muscles s'est faite tout à fait normalement pour 39 animaux sur 40 ; un seul $\mathrm{pH}$ supérieur à 6 est enregistré pour l'un des muscles étudiés chez une vache âgée du lot maigre ce qui explique les écarts significatifs (tableau 8).

\section{- Teneur en matière sèche et en lipides totaux}

Les teneurs en lipides totaux et en matière sèche d'une viande peuvent être interprétées globalement puisqu'il existe une très bonne liaison entre elles $(r=0,92$ pour chacun des deux muscles analysés pour les 40 animaux de l'essai 2). On observe évidemment des teneurs en matière sèche et en lipides totaux plus faibles $(\mathrm{P}<0,01)$ pour les muscles provenant des vaches abattues maigres: par exemple, 1,7\%
Tableau 7. Résultats des analyses sensorielles de l'essai 2. Notes moyennes de tendreté, jutosité et flaveur obtenues sur les muscles long dorsal (LD) et gros anconé (GA).

Tableau 8. Influence de l'âge, de l'engraissement et d'un anabolisant sur le $\mathrm{pH}$ et sur les teneurs en matière sèche et lipides des muscles long dorsal et rhomboïde thoracique (essai 2).

L'engraissement permet d'améliorer la tendreté, la jutosité et la flaveur de la viande, la tendreté restant toujours plus faible pour le lot de vaches âgées. 
Tableau 9. Influence de l'âge, de l'engraissement et d'un anabolisant sur les mesures de cisaillement et la teneur en collagène total du muscle long dorsal (essai 2).

\begin{tabular}{|c|c|c|c|c|c|c|c|c|}
\hline & \multicolumn{4}{|c|}{ Tous les animaux } & \multicolumn{4}{|c|}{ Animaux engraissés } \\
\hline & \multirow[b]{2}{*}{ effectif } & \multicolumn{3}{|c|}{ viande crue } & \multirow[b]{2}{*}{ effectif } & \multicolumn{2}{|c|}{ viande cuite } & \multirow{2}{*}{\begin{tabular}{|c|} 
collagène \\
total \\
(mg/g \\
frais)
\end{tabular}} \\
\hline & & $\begin{array}{c}F \\
(\mathrm{daN})\end{array}$ & $\begin{array}{c}W \\
(d J)\end{array}$ & $W / F$ & & $\begin{array}{c}\mathrm{F} \\
(\mathrm{daN})\end{array}$ & $\begin{array}{l}W \\
(d J)\end{array}$ & \\
\hline Moyenne générale & 40 & 1,61 & 0,67 & 0,43 & 33 & 3,01 & 1,80 & 4,27 \\
\hline $\begin{array}{l}\text { Age } \\
5 \text { ans } \\
11 \text { ans }\end{array}$ & $\begin{array}{l}21 \\
19\end{array}$ & $\begin{array}{c}n s \\
1,62 \\
1,60\end{array}$ & $\begin{array}{c}n s \\
0,66 \\
0,69\end{array}$ & $\begin{array}{c}n s \\
0,41 \\
0,45\end{array}$ & $\begin{array}{l}18 \\
15\end{array}$ & $\begin{array}{c}n s \\
2,95 \\
3,09\end{array}$ & $\begin{array}{c}n s \\
1,76 \\
1,85\end{array}$ & $\begin{array}{c}n s \\
4,20 \\
4,36\end{array}$ \\
\hline $\begin{array}{l}\text { Engraissement } \\
\text { - maigre } \\
\text { - sans anabolisant } \\
\text { - avec anabolisant }\end{array}$ & $\begin{array}{r}7 \\
15 \\
18\end{array}$ & $\begin{array}{l}{ }^{* *} \\
2,04^{\prime \prime} \\
1,55^{b} \\
1,50^{b}\end{array}$ & $\begin{array}{l}\star * \\
0,68 \\
0,64 \\
0,70\end{array}$ & $\begin{array}{l}{ }^{* *} \\
0,33^{\mathrm{c}} \\
0,42^{\mathrm{h}} \\
0,47^{\mathrm{a}}\end{array}$ & $\begin{array}{l}- \\
15 \\
18\end{array}$ & $\begin{array}{c}n s \\
- \\
3,12 \\
2,92\end{array}$ & $\begin{array}{c}n s \\
- \\
1,83 \\
1,77\end{array}$ & $\begin{array}{c}n s \\
- \\
4,30 \\
4,25\end{array}$ \\
\hline $\begin{array}{l}\text { Age } \times \text { engraissement } \\
5 \text { ans : }\end{array}$ & & $n s$ & $n s$ & $n S$ & & $n s$ & $n s$ & $n s$ \\
\hline - maigre & 3 & 1,93 & 0,62 & 0,32 & - & - & - & - \\
\hline - sans anabolisant & 9 & 1,59 & 0,66 & 0,41 & 9 & 3,12 & 1,83 & 4,17 \\
\hline - avec anabolisant & 9 & 1,54 & 0,67 & 0,43 & 9 & 2,77 & 1,68 & 4,22 \\
\hline 11 ans : & 4 & 212 & 072 & 0.34 & _. & _- & _- & - \\
\hline - sans anabolisant & 6 & 1,49 & 0,62 & 0,43 & 6 & 3,12 & 1,83 & 4,50 \\
\hline - avec anabolisant & 9 & 1,45 & 0,73 & 0,51 & 9 & 3,06 & 1,86 & 4,27 \\
\hline
\end{tabular}

* * significatif au seuil de $1 \%, n s$ non significatif

a, b, $c$ : les valeurs suivies de lettres différentes sont significativement différentes.

de lipides dans le muscle long dorsal des animaux maigres contre $3,8 \%$ pour les vaches engraissées sans implant (tableau 8).

Les écarts d'âge des animaux n'entraînent pas de modification du gras intramusculaire. En revanche, la viande des animaux qui ont reçu des anabolisants est aussi - voire plus grasse - pour le muscle long dorsal que celle des animaux témoins. Ceci s'explique vraisemblablement par l'allongement de la durée d'engraissement des animaux implantés.

En réalité, ces variations de la teneur en lipides intramusculaires selon les différents facteurs étudiés sont tout à fait parallèles à celles de la proportion de tissu adipeux dans la carcasse. Le coefficient de corrélation linéaire entre ces deux paramètres est de 0,83 pour le muscle long dorsal et de 0,71 pour le muscle rhomboïde thoracique.

\section{- Force et travail de cisaillement}

L'âge n'a aucun effet significatif sur l'ensemble des mesures de cisaillement réalisées sur viande crue ou après chauffage pour les deux muscles étudiés (tableaux 9 et 10).

Chez les animaux maigres, la force de cisaillement mesurée sur viande crue, est toujours plus élevée $(\mathrm{P}<0,01)$ que chez les vaches engraissées avec ou sans implant pour les deux muscles considérés, avec par ailleurs, un rapport W/F significativement plus faible.

Chez les vaches engraissées, aucun écart significatif des mesures de cisaillement n'est mis en évidence suite à l'administration de l'acétate de trenbolone. On remarque, toutefois, que pour les deux muscles étudiés, le rapport
W/F est significativement plus élevé pour le lot ayant reçu l'implant.

\section{- Teneur en collagène total}

Chez les vaches engraissées, la teneur en collagène total $\mathrm{du}$ muscle long dorsal est en moyenne de 4,27 mg par g de viande fraîche. Elle ne diffère pas significativement avec l'âge des animaux ou l'administration d'acétate de trenbolone. Ce dosage n'a pas été fait chez les animaux maigres (tableau 9).

\section{- Pouvoir de rétention d'eau de la viande}

Le pouvoir de rétention d'eau de la viande, apprécié par la perte de poids à la pression ou à la cuisson, ne varie pas avec les différents facteurs étudiés (âge, état d'engraissement, anabolisant) (tableau 11).

\section{- Caractéristiques de couleur}

Il n'existe pas de modifications significatives des teneurs en fer héminique des muscles étudiés selon l'âge, l'état d'engraissement ou l'utilisation d'un anabolisant. On note un pourcentage de lumière réfléchie plus faible $(\mathrm{P}<0,01)$ chez les animaux maigres qui peut être facilement relié au $\mathrm{pH}$ moyen plus élevé pour ce lot d'animaux (tableau 11).

\section{c / Effets des différents facteurs analysés sur la qualité de la viande}

\section{- Effet de l'âge}

Pour une même teneur en lipides intramusculaires, de l'ordre de 3,7 \% dans le long dorsal de la $6^{\text {e }}$ côte, la viande des vaches les plus jeunes ( 5 ans) est jugée plus tendre que celle 


\begin{tabular}{|c|c|c|c|c|c|c|c|}
\hline & \multicolumn{4}{|c|}{ Tous les animaux } & \multicolumn{3}{|c|}{ Animaux engraissés } \\
\hline & \multirow[b]{2}{*}{ effectif } & \multicolumn{3}{|c|}{ viande crue } & \multirow[b]{2}{*}{ effectif } & \multicolumn{2}{|c|}{ viande cuite } \\
\hline & & $\begin{array}{c}F \\
(\mathrm{daN})\end{array}$ & $\begin{array}{l}W \\
\text { (d) })\end{array}$ & $\mathrm{W} / \mathrm{F}$ & & $\begin{array}{c}\mathrm{F} \\
(\mathrm{daN})\end{array}$ & $\begin{array}{l}W \\
\text { (dJ) }\end{array}$ \\
\hline Moyenne générale & 40 & 3,47 & 1,27 & 0,38 & 33 & 3,61 & 2,05 \\
\hline $\begin{array}{l}\text { Age } \\
5 \text { ans } \\
11 \text { ans }\end{array}$ & $\begin{array}{l}21 \\
19\end{array}$ & $\begin{array}{c}n s \\
3,47 \\
3,46\end{array}$ & $\begin{array}{c}n s \\
1,31 \\
1,23\end{array}$ & $\begin{array}{c}n s \\
0,38 \\
0,37\end{array}$ & $\begin{array}{l}18 \\
15\end{array}$ & $\begin{array}{c}n s \\
3,49 \\
3,75\end{array}$ & $\begin{array}{c}n s \\
1,99 \\
2,12\end{array}$ \\
\hline $\begin{array}{l}\text { Engraissement } \\
\text { - maigre } \\
\text { - sans anabolisant } \\
\text { - avec anabolisant }\end{array}$ & $\begin{array}{r}7 \\
15 \\
18\end{array}$ & $\begin{array}{c}* * \\
4,80^{\mathrm{a}} \\
3,32^{\mathrm{b}} \\
3,08^{\mathrm{b}}\end{array}$ & $\begin{array}{c}n s \\
1,36 \\
1,20 \\
1,29\end{array}$ & $\begin{array}{l}* * \\
0,28^{\mathrm{c}} \\
0,37^{\mathrm{b}} \\
0,42^{\text {a }}\end{array}$ & $\begin{array}{l}- \\
15 \\
18\end{array}$ & $\begin{array}{c}n s \\
- \\
3,53 \\
3,67\end{array}$ & $\begin{array}{c}n s \\
- \\
1,99 \\
2,10\end{array}$ \\
\hline $\begin{array}{l}\text { Age } \times \text { engraissement } \\
5 \text { ans : }\end{array}$ & & $n s$ & $n s$ & $n s$ & & $n s$ & $n s$ \\
\hline - maigre & 3 & 4,91 & 1,58 & 0,32 & - & - & - \\
\hline - sans anabolisant & 9 & 3,31 & 1,20 & 0,37 & 9 & 3,38 & 1,90 \\
\hline - avec anabolisant & 9 & 3,16 & 1,32 & 0,42 & 9 & 3,60 & 2,08 \\
\hline 11 ans : & & & & & & & \\
\hline - maigre & 4 & 4,72 & 1,20 & 0,25 & - & - & - \\
\hline $\begin{array}{l}\text { - sans anabolisant } \\
\text { - avec anabolisant }\end{array}$ & $\begin{array}{l}6 \\
9\end{array}$ & $\begin{array}{l}3,33 \\
3,00\end{array}$ & $\begin{array}{l}1,20 \\
1,26\end{array}$ & $\begin{array}{l}0,37 \\
0,43\end{array}$ & $\begin{array}{l}6 \\
9\end{array}$ & $\begin{array}{l}3,76 \\
3,74\end{array}$ & $\begin{array}{l}2,12 \\
2,12\end{array}$ \\
\hline
\end{tabular}

Tableau 10. Influence de l'âge, de l'engraissement et d'un anabolisant sur les mesures de cisaillement du muscle rhomboïde thoracique (essai 2).

** significatif au seuil de $1 \%, n s$ non significatif

$\mathrm{a}, \mathrm{b}, \mathrm{c}$ : les valeurs suivies de lettres différentes sont significativement différentes.

\begin{tabular}{|c|c|c|c|c|c|c|c|}
\hline \multirow[b]{2}{*}{ Muscle (1) } & \multicolumn{2}{|c|}{$\begin{array}{l}\text { Perte de poids } \\
\text { à la pression }(\%) \\
\text { (Tous les animaux) }\end{array}$} & \multicolumn{2}{|c|}{$\begin{array}{c}\text { Perte de poids } \\
\text { à la cuisson (\%) } \\
\text { (Animaux engraissés) }\end{array}$} & \multicolumn{2}{|c|}{$\begin{array}{l}\text { Fer héminique } \\
\text { ( } \mu \text { g/g frais) } \\
\text { (Tous les animaux) }\end{array}$} & \multirow{2}{*}{$\begin{array}{c}\text { Lumière } \\
\text { réfléchie } \\
\text { (\%) } \\
\text { (Tous les } \\
\text { animaux) } \\
\text { LD }\end{array}$} \\
\hline & LD & RT & LD & RT & LD & RT & \\
\hline Moyenne générale & 16,9 & 17,0 & 14,5 & 12,0 & 18,6 & 21,0 & 41,7 \\
\hline $\begin{array}{l}\text { Age } \\
5 \text { ans } \\
11 \text { ans }\end{array}$ & $\begin{array}{c}n s \\
16,7 \\
17,1\end{array}$ & $\begin{array}{c}n s \\
17,0 \\
17,0\end{array}$ & $\begin{array}{c}n s \\
14,5 \\
14,4\end{array}$ & $\begin{array}{c}n s \\
11,5 \\
12,6\end{array}$ & $\begin{array}{c}n s \\
19,1 \\
18,1\end{array}$ & $\begin{array}{c}n s \\
21,2 \\
20,8\end{array}$ & $\begin{array}{c}n s \\
43,6 \\
39,6\end{array}$ \\
\hline $\begin{array}{l}\text { Engraissement } \\
\text { - maigre } \\
\text { - sans anabolisant } \\
\text { - avec anabolisant }\end{array}$ & $\begin{array}{c}n s \\
17,2 \\
16,1 \\
17,5\end{array}$ & $\begin{array}{c}n s \\
15,7 \\
16,7 \\
17,8\end{array}$ & $\begin{array}{c}n s \\
- \\
14,8 \\
14,2\end{array}$ & $\begin{array}{c}n s \\
- \\
11,7 \\
12,3\end{array}$ & $\begin{array}{c}n s \\
16,5 \\
18,7 \\
19,4\end{array}$ & $\begin{array}{c}n s \\
20,3 \\
20,6 \\
21,6\end{array}$ & $\begin{array}{l}* * \\
29,9^{\mathrm{b}} \\
45,2^{\mathrm{a}} \\
43,3^{\mathrm{a}}\end{array}$ \\
\hline $\begin{array}{l}\text { Age } \times \text { engraissement } \\
5 \text { ans : }\end{array}$ & $n s$ & $n s$ & $n s$ & $n s$ & $n s$ & $n s$ & $n s$ \\
\hline $\begin{array}{l}\text { - maigre } \\
\text { - sans anabolisant } \\
\text { - avec anabolisant }\end{array}$ & $\begin{array}{l}17,3 \\
16,7 \\
16,5\end{array}$ & $\begin{array}{l}16,6 \\
16,6 \\
17,4\end{array}$ & $\begin{array}{c}- \\
15,2 \\
13,9\end{array}$ & $\begin{array}{c}- \\
11,1 \\
11,9\end{array}$ & $\begin{array}{l}17,9 \\
19,2 \\
19,4\end{array}$ & $\begin{array}{l}20,5 \\
20,9 \\
21,7\end{array}$ & $\begin{array}{l}30,7 \\
49,0 \\
42,5\end{array}$ \\
\hline $\begin{array}{l}11 \text { ans : } \\
\text { - maigre } \\
\text { - sans anabolisant } \\
\text { - avec anabolisant }\end{array}$ & $\begin{array}{l}17,1 \\
15,1 \\
18,5\end{array}$ & $\begin{array}{l}15,0 \\
16,8 \\
18,1\end{array}$ & $\begin{array}{l}- \\
14,0 \\
14,4\end{array}$ & $\begin{array}{c}- \\
12,5 \\
12,7\end{array}$ & $\begin{array}{l}15,5 \\
17,9 \\
19,4\end{array}$ & $\begin{array}{l}20,2 \\
20,2 \\
21,6\end{array}$ & $\begin{array}{l}29,2 \\
39,6 \\
44,2\end{array}$ \\
\hline
\end{tabular}

Tableau 11. Pouvoir de rétention d'eau et caractéristiques de couleur de la viande (essai 2).

(1) LD : Long dorsal ; RT : Rhomboïde thoracique

a, b, $\mathrm{c}$ : les valeurs suivies de lettres différentes sont significativement différentes.

$\star *$ significatif au seuil de $1 \%$, ns non significatif.

des animaux les plus âgés (11 ans). Cet écart de tendreté à la dégustation ne s'accompagne pas de différences significatives des résultats de cisaillement. L'évolution de la force de cisaillement avec l'âge des animaux n'est pas toujours facile à mettre en évidence. Souvent, elle ne concerne pas tous les muscles d'une carcasse ; elle est de faible amplitude et peut avoir une évolution non linéaire. Dryden et al (1979), en étudiant 8 muscles par carcasse chez des vaches de race Hereford âgées de 3, 6 et 10 ans, abattues maigres, montrent que seul le muscle long dorsal présente des variations significatives avec l'âge des résultats de cisaillement obtenus sur viande cuite; la valeur obtenue à 6 ans étant paradoxalement plus faible que celles obtenues à 3 ou 10 ans.

Les teneurs en collagène total mesurées sur le muscle long dorsal ne sont pas différentes 
selon l'âge des vaches. Ce résultat est conforme à la bibliographie, où l'on ne trouve aucune indication d'une augmentation systématique du collagène musculaire avec l'âge ; par contre, la stabilité thermique du collagène a tendance à augmenter lorsque l'animal vieillit. Toutefois, des mesures de solubilité du collagène réalisées par Culioli et al (1990) sur le muscle long dorsal des animaux engraissés de l'essai 2 ayant fait l'objet de l'analyse sensorielle n'apparaissent pas significativement différentes selon l'âge des animaux. Dans le cas précis de cette étude de l'effet de l'âge sur la tendreté de la viande, l'analyse sensorielle apparaît comme une méthode plus sensible que le cisaillement ou les dosages de collagène. Cependant, les mesures de cisaillement et les dégustations ne sont pas effectuées au même stade de maturation de la viande.

La jutosité et la flaveur de la viande appréciées par le jury de dégustateurs comme le pouvoir de rétention d'eau mesuré au laboratoire ne sont pas altérés significativement par le vieillissement de l'animal. Les caractéristiques de couleur sont, elles aussi, indépendantes de l'âge entre 5 et 11 ans.

\section{- Effet de l'état d'engraissement des carcasses}

Dans les deux essais, la viande des animaux maigres $(1,7 \%$ de lipides intramusculaires pour le long dorsal contre $4 \%$ environ chez les vaches engraissées) est toujours jugée plus dure à la dégustation. Cela s'accompagne d'une force de cisaillement mesurée sur le produit cru significativement plus élevée pour les deux muscles étudiés. La viande des animaux maigres est également jugée de moindre jutosité et de flaveur moins intense. Ces observations rejoignent en partie celles de Dryden et al (1979) qui montrent que l'effet d'une réalimentation faisant passer la teneur en lipides de la carcasse de $12,7 \%$ à $25 \%$ chez des vaches Hereford âgées de 7 à 12 ans entraîne, pour certains muscles, une amélioration des notes de tendreté à la dégustation sans qu'il y ait systématiquement une variation des résultats de cisaillement. Par rapport aux animaux maigres, une diminution de la valeur de cisaillement mesurée sur long dorsal est en revanche observée après 56 jours ou 84 jours d'engraissement chez des vaches Hereford et Angus de 9 à 13 ans (Matulis et al 1984). Cette moins bonne tendreté de la viande des carcasses maigres peut souvent être expliquée, au moins partiellement par une sensibilité à la contracture au froid plus importante suite au manque de gras de couverture. En principe, ce phénomène ne devrait pas s'être produit dans nos essais compte tenu du régime de froid appliqué.

\section{- Effet de l'acétate de trenbolone}

L'implant d'acétate de trenbolone n'a entraîné aucune modification significative des notations de l'analyse sensorielle ou des caractéristiques physico-chimiques à l'exception de la teneur en lipides intramusculaires. Ces observations ne confirment pas les résultats obtenus chez des boeufs Charolais pour lesquels, un effet négatif de l'anabolisant sur la tendreté de la viande est mis en en évidence (Touraille 1986, Bordes et al 1988). Plusieurs éléments d'explication peuvent aider à comprendre l'absence d'effet marqué de l'anabolisant :

- les animaux implantés ont été abattus tardivement, 90 jours après la pose de l'implant,

- l'état d'engraissement à l'abattage des animaux implantés était égal à celui des témoins voire supérieur et un peu excessif,

- l'écart de reprise de poids dû au facteur de croissance était limité surtout pour le lot de vaches jeunes,

- les effectifs de vaches dont les muscles ont fait l'objet d'analyses sensorielles étaient faibles.

\section{Conclusion}

La remise "en état» des vaches de réforme de race Charolaise s'avère intéressante puisqu'elle permet d'améliorer la qualité des carcasses mais surtout les qualités organoleptiques de la viande. Cette finition peut être de courte durée (60 à 70 jours) avec une ration très énergétique à base d'ensilage de maïs.

Dans le cas de ces deux essais, les vaches ne recevant pas d'anabolisant ont alors en moyenne un gain de poids vif journalier de 1100 à $1200 \mathrm{~g}$, qui est vraisemblablement sousévalué compte-tenu des variations du poids du contenu digestif observées en cours d'engraissement. Leur gain de carcasse, pouvant dépasser $60 \mathrm{~kg}$, est composé de 37 à $42 \%$ de muscle.

La prolongation de l'engraissement au delà de 70 jours ne paraît pas très utile puisque le surplus de carcasse ainsi obtenu est essentiellement composé de gras.

Les performances des animaux les plus âgés (11 ans en moyenne) restent correctes mais inférieures à celles des jeunes $(5$ ans en moyenne) en ce qui concerne l'efficacité alimentaire, le rendement à l'abattage et surtout la tendreté de la viande. Il faut remarquer que le schéma expérimental utilisé ne permet pas de déterminer à partir de quel âge exactement les performances et la tendreté de la viande diminuent. Il serait pourtant intéressant de le connaître plus précisement pour la définition des conditions de production d'animaux de qualité.

L'utilisation de l'acétate de trenbolone permet d'améliorer les performances des animaux avec, dans le cas de cet essai, une très bonne réponse des vaches les plus âgées. Aucun effet négatif de l'anabolisant sur les caractéristiques physico-chimiques ou sur les qualités organoleptiques de la viande n'a pu être mis en évidence.

\section{Remerciements}

Cette étude a été réalisée grâce au soutien financier du Conseil Régional de Bourgogne et de la DGER du Ministère de l'Agriculture.

Les auteurs tiennent à remercier toutes les personnes qui ont contribué au bon déroulement de l'expérimentation, à la collecte et au traitement des données : J.P. Blanchon, Directeur du Domaine Expérimental INRA d'Epoisses; F. Delamarche, M. Des- 
gouilles, F. Faurie, Y. Grospiron et B. Guérin, techniciens au Laboratoire de la Chaire de Zootechnie de l'ENSSAA de Dijon; V. Allard, V. Ponchelet et M-J. Kempf, pour les travaux de secrétariat et de dactylographie ; J.P. Argaud et P. Penichon, élèves de l'ENITA de Dijon qui ont réalisé leur mémoire d'études dans le cadre de ce programme; G. Cuylle, R. Jailler et Y. Vantomme du Laboratoire Croissance et Métabolismes des Herbivores de l'INRA de Theix et M-C. Bayle de la Station de Recherche sur la Viande de I'INRA de Theix, leur aide a toujours été précieuse.

Enfin, il faut remercier particulièrement D. Contour, technicien au Domaine INRA de Dijon-Epoisses qui a suivi au jour le jour les animaux de ces essais.

\section{Références bibliographiques}

AGABRIEL J., GIRAUD J.M., PETIT M., 1986. Détermination et utilisation de la note d'engraissement en élevage allaitant. Bull. Tech. CRZV Theix, INRA, 66, 43-50.

BERANGER C., MALTERRE C., 1968. Influence d'un stéroïde triénique à activité anabolisante sur l'engraissement de vaches taries. Compte rendu des séances de la Société de Biologie. Tome 162 (5-6), 1157-1164.

BERANGER C., NEGRIN M., MALTERRE C., 1970. Evolution du gain de poids vif et de l'état d'engraissement des vaches taries au pâturage. Ann. Zootech., 19, 53-66.

BONNET M., KOPP J., 1984. Dosage du collagène dans les tissus conjonctifs, la viande et les produits carnés. Cah. Techn. INRA. 5, 19-30.

BORDES P. BOCCARD R. RENERRE M., TOURAILLE C., BAYLE M.C., FOURNIER R. LABAS R., 1988. Viande de boeuf sous label. Comparaisons entre des caractéristiques qualitatives des viandes de boeufs Charolais témoins labels et implantés. Viandes et produits carnés, 9, 239240.

CEREOPA, 1987. Situation et perspectives d'évolution des productions de viande clans le Centre Est. Livres I, II et IV. Ed. CEREOPA, 16 rue Claude Bernard, Paris.

CHILLIARD Y., ROBELIN I., REMOND B., 1984. In vivo estimation of body lipid mobilization and reconstitution in dairy cattle. Can. J. Anim. Sci., 64 (Supl.), 236-237.

COLLEAU J.J., MALTERRE: C., TOURAILLE C., 1984. Influence du type génétique et du niveau de production laitière sur la qualité des carcasses et des viandes de vaches laitières réformées. Bull. Tech. CRZV Theix, INRA, 58, 45-52.

CULIOLI J, BORDES P., DUMONT R, 1990. Influence of a trenbolone acetate implant on the composition and the ageing kinetics of the longissimus dorsi muscle of cull cows. Sciences des aliments, 10, 533-542.

DOREAU M., ROBELIN J., LESTRADE A., 1985. Effects of physiological state and body fatness on digestive tract weight and composition in the dairy cow. Livest. Prod. Sci., 12, 379-385.

DRYDEN F.D., MARCHELLO I.A., TINSLEY A., MARTINS C.B.. WOOTEN R.A., ROUBICEK C.B., SWINGLE R.S., 1979. Acceptability of selected muscles from poor condition and realimented cull range cows. J. Food. Sci. 44, 1058-1062.

FOLCH J., LEES M., SLOANE STANL.EY G.H., 1957. A simple method for the isolation and purification of total lipides from animal tissues. J. Biol. Chem., 226, 497-509. FREBLING J., POUJARDIEU B., VISSAC B., BERANGER C. TEISSIER J.H., 1967. Station de sélection bovine. Compte rendu technique n" 1 . Note générale. BTI, 225, $887-894$

GEAY Y., 1986. Emploi des additifs alimentaires et des anabolisants pour la production de viande de gros bovins. 2. Intérêt zooterhnique des substances à activités hormonales. In D. Micol éd : Production de Viande bovine. INRA Paris, pp. $422-434$

GOUTEFONGEA R., 1963. Comparaison de différentes méthodes de mesure du pouvoir de rétention d'eau de la viande de porc. Liaison avec le pH. Ann. Zootechn., 12, 125-132

HORNSEY H.C., 1956. The colour of cooked cured pork. I. Estimation of the nitric oxide-haem pigments. J. Sci. Food. Agric., 7, 534-540.
ITEB, 1989. Lait et viande bovine en 1988. Perspectives 1989. ITEB Publications, 149 rue de Bercy, Paris.

JONES S.D.M., 1981. Offal growth in young and mature dairy cows. Can. J. Anim. Sci., 61, 607-611.

JONES S.D.M., MACLEOD G.K., 1981. The feedlot performance and carcass composition of young and mature cull Holstein cows. Can. J. Anim. Sci., 61, 593-599.

JONES S.D.M., 1983. Tissue growth in young and mature cull Holstein cows fed a high energy diet. J. Anim. Sci., $56,64-70$

LHERM M., BEBIN D., LIENARD G., 1988. Orientation des productions bovines charolaises en Creuse. INRA Prod. Anim., 1, 97-109.

MALTERRE C., 1972. Peut-on améliorer l'engraissement de vaches laitières réformées? in L'Elevage N" hors série "L'exploitation moderne du troupeau laitier". 141-150.

MALTERRE C., 1986. Production de viande de vaches de réforme. In D. Micol éd : Production de Viande bovine, INRA, Paris, 247-269.

MALTERRE C., JONES S.D.M. 1991. Meat production from heifers and cull cows. In Beef Cattle Production. Ed. R. Jarrige, Elsevier (sous presse).

MALTERRE C., ROBELIN J., AGABRIEL J., BORDES P., 1989. Engraissement des vaches de réforme de race Limousine. INRA Prod. Anim., 2, 325-334.

MATULIS R.J., McKEITH F.K., FAULKNER D.B., BERGER L.L., GEORGE P., 1987. Growth and carcass characteristics of cull cows after different times-on-feed. J. Anim. Sci. $65,669-674$

ROBELIN J., AGABRIEL I., 1986. Estimation de l'état d'engraissement des bovins vivants à partir de la taille des cellules adipeuses. Bull. Tech. CRZV Theix, INRA, $66,37-41$.

ROBELIN I, AGABRIEL I MALTERRE C, BONNEMAIRE J., 1990. Changes in body composition of mature dry cows of Holstein Limousine and Charolaise breeds during fattening. I: Skeleton muscles fatty tissues and offal. Livest. Prod. Sci., 25, 199-215.

SALE P., 1971. Evolution de quelques propriétés mécaniques du muscle pendant la maturation. Bull. Tech. CRZV Theix INR $\Lambda$. 6, 35-44.

SMITH N.E., BALDWIN R.L., 1974. Effects of beef pregnancy and lactation on weight of organs and tissues in dairy cattle. J. Dairy Sci., 57, 1055-1060.

SWINGLE R.S., ROUBICEK C.B., WOOTEN R.A., MARCHELLO J.A., DRYDEN F.D., 1979. Realimentation of cull range cows. I. Effect of final body condition an dietary energy level on rate, efficiency and composition of gains. I. Anim. Sci., 48, 913-918.

TOURAILLE C., 1986. Conséquences de l'emploi des anabolisants sur la qualité de la viande. In D. Micol éd : Production de Viande bovine, INRA Paris, pp 445-449.

WOOTEN R.A., ROUBICEK C.B., MARCHELLO J.A.. DRYDEN F.D., SWINGLE R.S., 1979. Realimentation of cull range cows. 2. Changes in carcass traits. J. Anim. Sci., 48, 823-830. 


\section{Summary}

\section{The fattening of Charolais cull cows}

Two experiments were conducted to investigate the effects of length of the feeding period (0, 70 or 109 days), age ( 5 or 11 years) and use of trenbolone acetate on liveweight, dry matter intake, carcass weight, carcass compositional changes and meat quality of 56 cull Charolais cows fed on silage. A total of 11 additional cows were slaughtered at the beginning of the feeding period to estimate initial body composition.

During the $\mathbf{7 0}$ day feeding period, cows without the trenbolone acetate implant had a daily liveweight gain of $1.2 \mathrm{~kg}$. The estimated daily dry matter intake was $13.7 \mathrm{~kg}$, their feed efficiency was $90 \mathrm{~g}$ of gain per UFL ingested. The average warm carcass weight of fed cows was $385 \mathrm{~kg}$ and consisted on average of $66 \%$ meat, $19 \%$ fat and $15 \%$ bone. Carcass weight gains averaged $63 \mathrm{~kg}$ for Experiment 1 with a muscle content of $37 \%$.

An extended fattening period of 110 days resulted in an increased carcass weight ( 20 to $30 \mathrm{~kg}$ ), but this consisted mostly of fat.

In this experiment, there was no evidence of any negative effects of age on the level of performance during fattening. Even though, the liveweight gain, feed efficiency and the per- centage yield at slaughter tended to decrease for 11 year old cows.

Implantation with trenbolone acetate resulted in a greater liveweight gain of $57 \mathrm{~kg}$ and in an increase in the lean proportion of the carcass gain $(47 \%$ versus $41 \%$ for the control group of the 11 year old cows). The fattening period was increased 27 days in this instance.

Acceptability of the meat, as measured by tenderness, juiciness and flavour, increased for cows which were realimented, while shear mesurements decreased. Significant differences in tenderness with advancing age were obtained in the two muscles studied (values of 4.99 for old cows versus 5.45 for young cows). The age of the animal had no influence on the level of juiciness, flavour, shear measurments, pigments, water holding and intramuscular fat. No significant negative effects were determined for the trenbolone implant for any of the meat traits evalued.

DUMONT R., ROUX M., AGABRIEL J., TOURAILLE C., BONNEMAIRE J., MALTERRE C. et ROBELIN J. 1991. Engraissement des vaches de réforme de race charolaise. Facteurs de variation des performances zootechniques, de la composition tissulaire des carcasses et de la qualité organoleptique de la viande. INRA Prod. Anim., 4 (4), 271 - 286. 